\title{
Functional imaging of mitochondria in retinal diseases using flavoprotein fluorescence
}

\author{
Andrew X. Chen ${ }^{1,2,3} \cdot$ Thais F. Conti $\mathbb{1}^{2,3} \cdot$ Grant L. Hom ${ }^{2,3} \cdot$ Tyler E. Greenlee $\mathbb{1}^{2,3} \cdot$ Raffaele Raimondi $\mathbb{1}^{2,3} \cdot$ \\ Isaac N. Briskin $\mathbb{1}^{4} \cdot$ Collin A. Rich ${ }^{5} \cdot$ Reecha Kampani $^{2} \cdot$ Robert Engel $^{2} \cdot$ Sumit Sharma ${ }^{1,2,3} \cdot$ Katherine E. Talcott $^{2,3}$. \\ Rishi P. Singh ${ }^{1,2,3}$
}

Received: 15 June 2020 / Revised: 1 July 2020 / Accepted: 16 July 2020 / Published online: 24 July 2020

(c) The Author(s), under exclusive licence to The Royal College of Ophthalmologists 2020

\begin{abstract}
Mitochondria are critical for cellular energy production and homeostasis. Oxidative stress and associated mitochondrial dysfunction are integral components of the pathophysiology of retinal diseases, including diabetic retinopathy (DR), agerelated macular degeneration, and glaucoma. Within mitochondria, flavoproteins are oxidized and reduced and emit a green autofluorescence when oxidized following blue light excitation. Recently, a noninvasive imaging device was developed to measure retinal flavoprotein fluorescence (FPF). Thus, oxidized FPF can act as a biomarker of mitochondrial dysfunction. This review article describes the literature surrounding mitochondrial FPF imaging in retinal disease. The authors describe the role of mitochondrial dysfunction in retinal diseases, experiments using FPF as a marker of mitochondrial dysfunction in vitro, the three generations of retinal FPF imaging devices, and the peer-reviewed publications that have examined FPF imaging in patients. Finally, the authors report their own study findings. Goals were to establish normative reference levels for FPF intensity and heterogeneity in healthy eyes, to compare between healthy eyes and eyes with diabetes and DR, and to compare across stages of DR. The authors present methods to calculate a patient's expected FPF values using baseline characteristics. FPF intensity and heterogeneity were elevated in diabetic eyes compared to age-matched control eyes, and in proliferative DR compared to diabetic eyes without retinopathy. In diabetic eyes, higher FPF heterogeneity was associated with poorer visual acuity. In conclusion, while current retinal imaging modalities frequently focus on structural features, functional mitochondrial imaging shows promise as a metabolically targeted tool to evaluate retinal disease.
\end{abstract}

\section{Search terms and databases used}

The PubMed database of the National Center for Biotechnology Information (NCBI) was searched for peerreviewed literature using the terms "flavoprotein fluorescence retina", "flavoprotein autofluorescence retina", and

Rishi P. Singh

SINGHR@ccf.org

1 Case Western Reserve University School of Medicine, Cleveland, OH, USA

2 Cole Eye Institute, Cleveland Clinic, Cleveland, OH, USA

3 Center for Ophthalmic Bioinformatics, Cole Eye Institute, Cleveland Clinic, Cleveland, OH, USA

4 Department of Quantitative Health Sciences, Cleveland Clinic, Cleveland, $\mathrm{OH}$, USA

5 OcuSciences Inc., Ann Arbor, MI, USA "mitochondrial dysfunction imaging retina", yielding 152 results. Abstracts were reviewed and relevant articles were selected. From these articles, cited publications were also selected if related to the topic of this review.

\section{Introduction}

Mitochondrial metabolic dysfunction has been implicated in the pathogenesis and progression of retinal diseases, including diabetic retinopathy (DR), age-related macular degeneration (AMD), and glaucoma [1, 2]. In these conditions, a major cause of mitochondrial dysfunction is oxidative stress, which is characterized by overproduction of reactive oxygen species (ROS) and impairment of antioxidant defence systems [3-8]. Oxidative stress can be measured in the form of mitochondrial flavoprotein fluorescence (FPF). Under oxidizing conditions, flavoproteins that are excited by blue light will emit green 


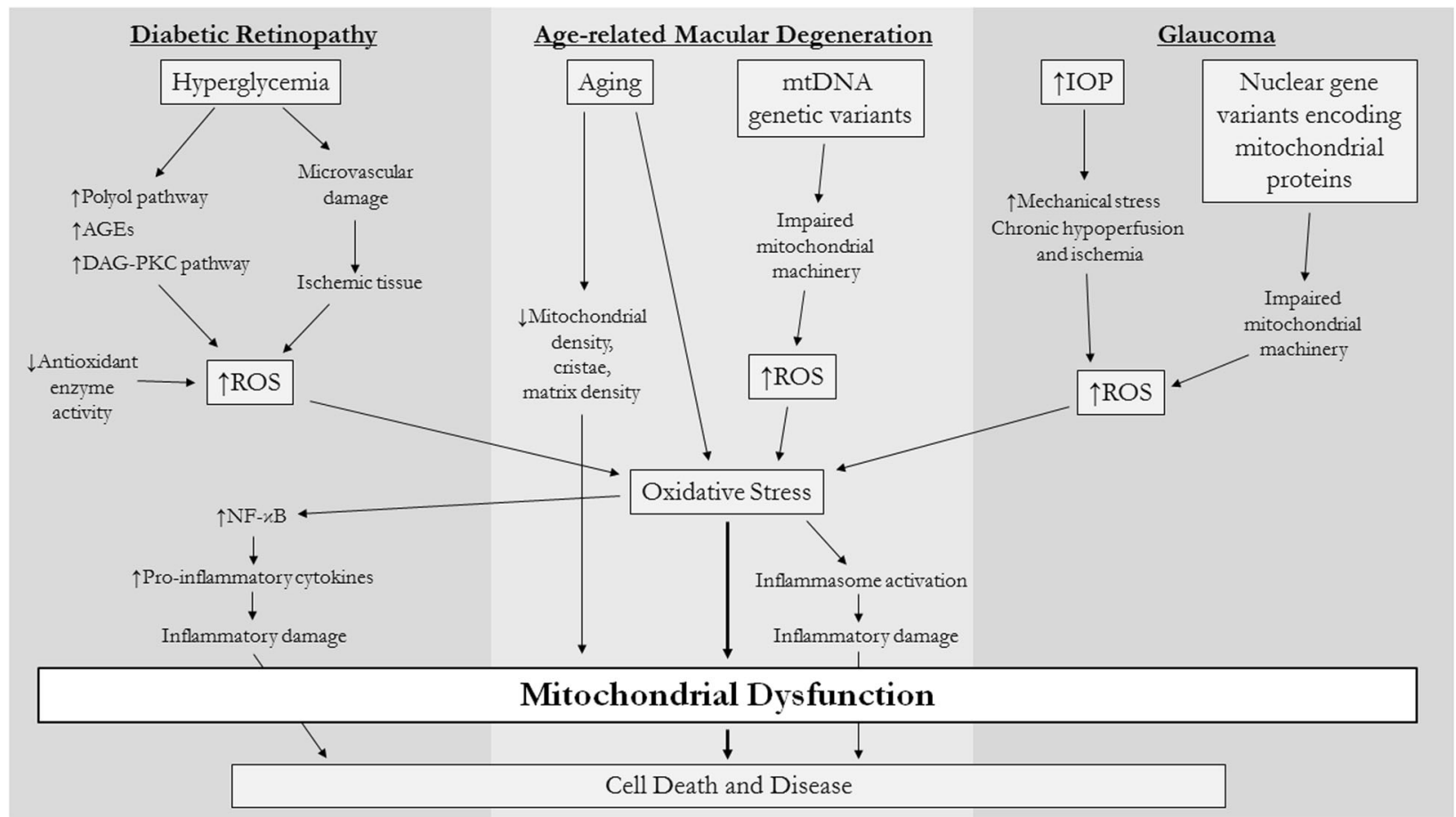

Fig. 1 Mechanisms of oxidative stress and mitochondrial dysfunction in retinal diseases. A number of complex cellular mechanisms are thought to contribute to reactive oxygen species (ROS) production in major retinal diseases. Oxidative stress is a shared feature of these conditions and leads to mitochondrial dysfunction and

autofluorescence $[9,10]$. Discovery of FPF led to the development of novel imaging devices that quantify FPF emitted from a patient's retina. Whereas most current imaging tests assess structural features of the retina, FPF assesses its metabolic function. Based on studies thus far, FPF has shown promise as a potential tool for screening, monitoring, and guiding treatment in patients with retinal disease [11-18].

The purpose of this review article is to summarize the current understanding of mitochondrial FPF imaging in retinal disease. Topics will include mitochondrial dysfunction and oxidative stress in retinal diseases, the molecular basis of FPF, and peer-reviewed studies on FPF imaging. The authors will also present their findings using the latest generation imaging device to study FPF in healthy control patients and patients with DR. Finally, the authors will discuss potential future directions for retinal FPF imaging.

\section{Mitochondrial dysfunction in retinal diseases}

\section{Overview}

Mitochondria are the primary energy-producing organelles of human cells. Through cellular respiration, they generate subsequent cell death. AGEs advanced glycation end products, $D A G$ $P K C$ diacylglycerol-protein kinase $\mathrm{C}, N F-\kappa B$ nuclear factor kappalight-chain-enhancer of activated $\mathrm{B}$ cells, $m t D N A$ mitochondrial DNA, $I O P$ intraocular pressure.

energy in the form of adenosine triphosphate (ATP). In addition, they coordinate numerous components of cellular metabolism, including apoptosis, regulation of cellular membrane potential, calcium signaling, steroid synthesis, hormone signaling, and immune signaling [19]. Mitochondrial function is essential to cellular homeostasis, and conversely, mitochondrial dysfunction leads to disruption of cellular processes and potential cell death.

Mitochondrial dysfunction has been identified as a key pathophysiologic process in normal aging and disease $[1,2]$. Tissues that are highly metabolically active, such as those of the brain, liver, and heart, are most susceptible to mitochondria-related damage. For instance, mitochondrial dysfunction has been highlighted as a key mechanism in neurodegenerative diseases, diabetes, and cerebral and cardiac ischemia-reperfusion injury [3].

The retina, another tissue with high metabolic needs, is also vulnerable to mitochondrial dysfunction. Mitochondria are essential for metabolic maintenance of both the neural retina and retinal pigment epithelium (RPE). In fact, photoreceptors have limited mitochondrial reserve capacity and are particularly susceptible to disruptions in energy homeostasis [20]. Numerous studies have demonstrated that mitochondria are implicated in pathophysiologic mechanisms underlying major ophthalmic conditions such as DR, AMD, and glaucoma [1, 2] (Fig. 1). 


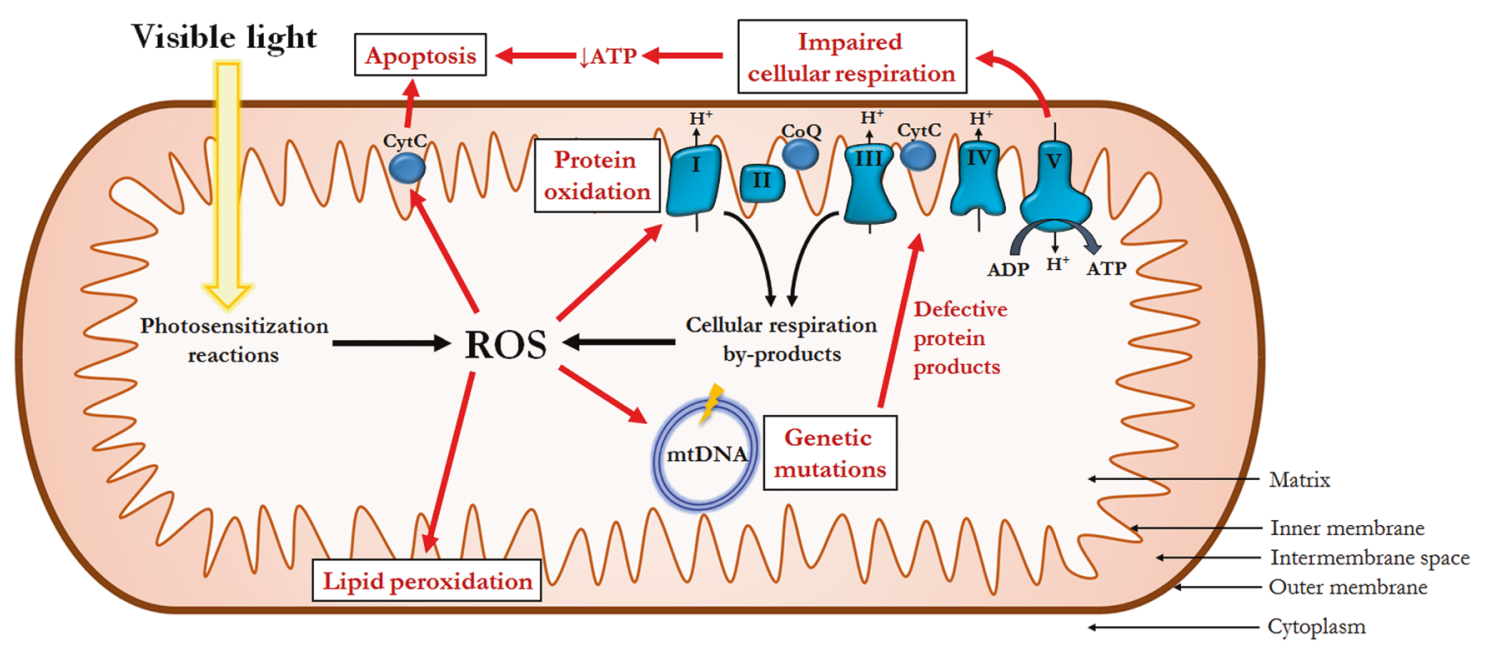

Fig. 2 Impact of oxidative stress on mitochondrial function. Under normal conditions, reactive oxygen species (ROS) are generated as a by-product of cellular respiration and via photosensitization reactions. Oxidative damage to mitochondria DNA (mtDNA) leads to the production of defective mtDNA-encoded proteins. Diminished adenosine triphosphate (ATP) production secondary to impaired cellular respiration and activation of cytochrome $\mathrm{c}(\mathrm{CytC})$ ultimately leads to apoptotis. Impairment of the electron transport chain also exacerbates baseline electron leak, which further increases ROS production. CoQ coenzyme Q, ADP adenosine diphosphate.
In each of these diseases, oxidative stress is the common pathway leading to mitochondrial dysfunction [8]. Oxidative stress is characterized by an imbalance between ROS and antioxidant defences of the cell, and leads to the damage and impairment of mitochondria. Mitochondrial damage secondary to oxidative stress plays a key role in major diseases, including ophthalmic disease [3-5].

Oxidative damage occurs through a variety of mechanisms (Fig. 2). ROS oxidize and alter the structure of mitochondrial macromolecules and enzymes. Mitochondrial DNA (mtDNA) is particularly susceptible to oxidative damage and resultant mutations due to relatively poor DNA repair mechanisms and an absence of protective histones and DNA-binding proteins [21]. Experiments have shown that human RPE cells placed under oxidative stress exhibit higher rates of damage to mtDNA and slower repair compared to nuclear DNA [22]. As a consequence of mtDNA damage, expression of critical mtDNA-encoded mitochondrial proteins is impaired.

In addition, the mitochondrion itself is a site of continuous ROS formation. ROS are by-products of the redox reactions involved in oxidative phosphorylation, particularly from electron transport chain complexes I and III. In tissues exposed to sunlight such as the retina, photosensitization reactions induce the generation of even more ROS. Finally, damage to the mitochondrial genome results in impaired structure and function of the electron transport chain protein subunits for which it codes. Resultant dysfunction of the electron transport chain worsens electron leak and ROS generation, further contributing to oxidative stress and fueling a vicious cycle of mitochondrial damage and dysfunction [23].

\section{Diabetic retinopathy}

In diabetes, hyperglycemia is the main driver for the development of DR. In retinal tissues, hyperglycemia upregulates production of ROS, such as superoxide and hydrogen peroxide $[24,25]$. It has been hypothesized that hyperglycemia induces oxidative stress through a variety of complex cellular pathways, including increased production of sorbitol via the polyol pathway, enhanced production of advanced glycation end products (AGEs), and activation of the diacylglycerolprotein kinase C (DAG-PKC) pathway [26]. Oxidative stress triggers a sequence of mitochondrial fragmentation, DNA damage, increased membrane potential heterogeneity, decreased oxygen consumption, and cytochrome c release [27]. Mitochondrial release of cytochrome $\mathrm{c}$ activates the caspase cascade and commits the cell to apoptosis.

Decreased expression and activity of antioxidant enzymes has also been linked to retinal neuron apoptosis in diabetes [28]. Microvascular damage and resultant ischemia further contribute to enhanced ROS production and diminished antioxidant defences [29]. Oxidative stress also triggers activation of nuclear factor kappa-light-chain-enhancer of activated $B$ cells $(\mathrm{NF}-\mathrm{\kappa B})$, which initiates the release of pro-inflammatory cytokines [30]. In contrast, antioxidant supplementation and overexpression of mitochondrial antioxidant enzymes has prevented the development of retinopathy in diabetic animal models [31].

\section{Age-related macular degeneration}

Oxidative stress and mitochondrial dysfunction, particularly in the RPE, have been strongly implicated in AMD. 
Advanced age is a strong risk factor for AMD. The mitochondrial theory of aging contends that ROS generation over one's lifetime causes cumulative damage to mitochondrial DNA and other macromolecules. Both advanced age and AMD have been associated with increased mtDNA damage and decreased repair in retinal tissue [23, 32]. Histopathologic studies have similarly shown that with older age, there are reductions of RPE mitochondrial density, cristae, and matrix density, which are even more pronounced in patients with AMD [33]. Increased mitochondrial lipid peroxidation, protein and DNA oxidation, have also been observed in retinas of patients with AMD, particularly in those with geographic atrophy (GA) [34]. Meanwhile, genetic studies have revealed that certain mtDNA haplogroups are associated with AMD and drusen phenotype, and that retinal mtDNA exhibits more deletions and rearrangements in patients with AMD compared to agematched controls $[1,35,36]$. Proteomic studies of the RPE have also demonstrated altered expression patterns of proteins responsible for mitochondrial membrane trafficking, protein folding, and apoptosis regulation that correlate with AMD stage [37, 38]. Furthermore, oxidative stress in RPE cells activates inflammasomes, which may contribute to deleterious inflammation in AMD [39].

Antioxidant pathways have also proven to be important in AMD, and present a target for potential therapies. In animal models, downregulation of manganese superoxide dismutase, a key antioxidant enzyme, triggered oxidative stress and led to RPE dysfunction, choroidal damage, and photoreceptor loss, recapitulating the pathologic components of non-exudative AMD [40]. On the other hand, antioxidant supplements have proven to slow disease progression and visual acuity (VA) loss in AMD and are the current standard of care for individuals with non-exudative AMD [41, 42].

\section{Glaucoma}

Numerous mitochondria are present in the retinal ganglion cells (RGCs) and optic nerve head, the major sites of glaucomatous damage [43]. Patients with primary openangle glaucoma (POAG) have higher rates of mtDNA mutations and decreased mitochondrial respiratory activity compared to age-matched controls [44]. In animals, elevated intraocular pressure (IOP), a primary feature of glaucoma, increases oxidative stress and reduces antioxidant defences [45]. Elevated hydrostatic pressure in human RGC cultures has also induced abnormal mitochondrial fission and loss of mitochondrial cristae and ATP [46]. Although the mechanisms are not fully understood, researchers have hypothesized that elevated pressure leads to increased ROS production secondary to mechanical stress or chronic hypoperfusion and ischemia [47]. In normal tension glaucoma, variants have been identified in nuclear genes encoding mitochondrial proteins [48].

In addition, restoration of mitochondrial antioxidant systems may be protective in glaucoma. For instance, overexpression of optic atrophy type 1 protein, which is involved in mitochondrial inner membrane regulation, inhibited RGC death in an animal model of glaucoma [49]. In addition, intravitreal transplant of stem cells has been observed to effectively donate functional mitochondria to RGCs and prevent RGC loss resulting from mitochondrial damage [50].

\section{FPF as a marker of mitochondrial dysfunction in the retina}

\section{Overview}

Flavoproteins, which are enzyme proteins containing either flavin adenine dinucleotide (FAD) or flavin mononucleotide, are essential components of normal mitochondrial function. The human genome encodes 90 flavoproteins, which are primarily located in the mitochondria [51]. A number of mitochondrial flavoproteins participate in redox reactions that are fundamental to cellular respiration.

Oxidized flavoproteins are a readily detectable marker of oxidative stress by virtue of their autofluorescence. Oxidized, but not reduced, flavoproteins display green autofluorescence when excited by blue light [10]. The ratio of oxidized flavoproteins to reduced flavoproteins increases in environments of oxidative stress [52]. Thus, FPF acts a marker of oxidative stress and mitochondrial dysfunction.

\section{Spectral properties of FPF}

After FPF was first identified, experiments were performed to characterize its spectral properties. In studies of nonocular tissues, flavoproteins were seen to exhibit peak excitation at $430-470 \mathrm{~nm}$ (blue light) and peak emission at $520-540 \mathrm{~nm}$ (green light), which is similar to the spectra of FAD [53, 54]. Later investigations identified FPF in retinal tissues [55]. Notably in the retina, potential confounding fluorescence must be considered due to the presence of other fluorophores in the fundus.

Lipofuscin has been identified as the primary retinal fluorophore, and its fluorescence is strongest in the RPE [55]. Lipofuscin shows peak excitation at $510 \mathrm{~nm}$ and peak emission at $620-630 \mathrm{~nm}$, and exhibits some degree of spectral overlap with flavoproteins (Fig. 3). In contrast, the minor fluorophores vitamin $\mathrm{A}$, nicotinamide adenine dinucleotide (NADH) and nicotinamide adenine dinucleotide phosphate (NADPH) are optimally excited at $300-360 \mathrm{~nm}$, far from the excitation peak of flavoproteins [10, 56]. 


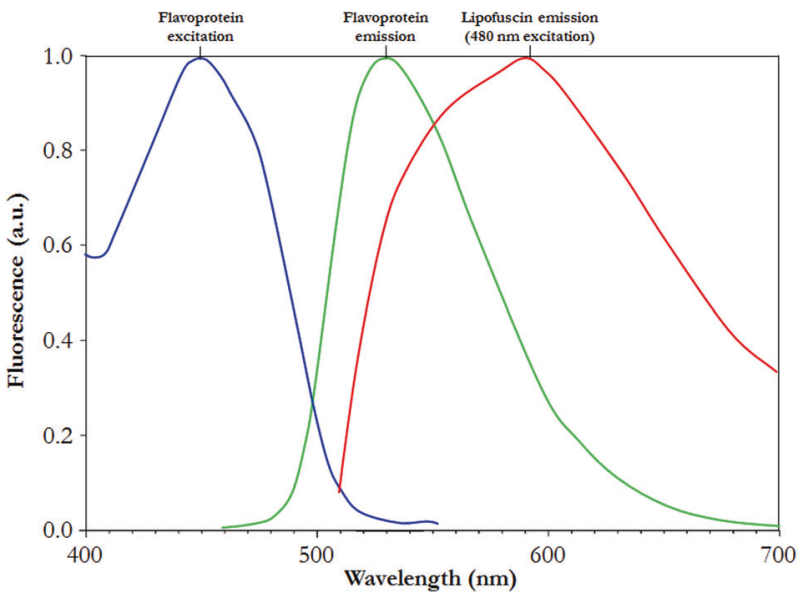

Fig. 3 Spectral properties of flavoproteins. Flavoprotein spectral curves are based on data from Islam et al. [98]. Lipofuscin emission (480 $\mathrm{nm}$ excitation) is based on data from Sparrow et al. [99]. Fluorescence is represented as arbitrary units (a.u.) and is normalized to peak intensity for each curve.

Melanin has been reported to fluoresce, but only weakly [57]. Bruch's membrane is also excited by ultraviolet wavelengths and emits blue-green fluorescence, which is consistent with elastin and collagen [58]. AGEs, which are lipid or protein molecules that are glycated due to exposure to sugars, have been linked to aging and development of degenerative diseases [59], and are predominantly excited by ultraviolet light and emit blue light.

In summary, the spectral properties of most retinal fluorophores are quite different from those of flavoproteins, which permits potential measurement of FPF with high signal-to-noise ratio. However, some degree of signal interference from a few fluorophores, namely fluorescein, must inform the selection of filters for an instrument that measures FPF.

Thus far, studies using FPF imaging devices in patients have provided evidence that filter bands placed near the emission peak of flavoproteins (and at the shoulder of lipofuscin emission) maximize signal-to-noise ratio, and that fluorescent signal is emitted primarily from flavoproteins, not lipofuscin or other fluorophores [11, 12, 60]. For example, FPF asymmetry has been identified between eyes in pseudotumor cerebri (PTC) and unilateral central serous chorioretinopathy (CSCR), which is unlikely to be due to significant asymmetric accumulation of other fluorophores $[18,60]$. Furthermore, in eyes with AMD, FPF hyperfluorescence was observed in areas of GA, where lipofuscin hypofluorescence is known to be present [13]. At the same time, it should be noted that lipofuscin is known to accumulate in drusen and has been linked to oxidative stress in AMD. Thus, in AMD, lipofuscin likely contributes both to increased levels of oxidized flavoproteins and potential contaminating autofluorescence [61].
Elevated FPF has also been observed in cultured neural retina and RPE cells only hours after exposure to oxidizing agents [11, 14]. These findings further link measured fluorescence to flavoproteins rather than lipofuscin or AGEs, since these fluorophores do not increase with acute exposure to oxidizing treatments, and because the tissues and recently passaged cells were too young to accumulate these products.

\section{Non-ocular studies and retinal studies in vitro}

In non-ocular tissues, studies have highlighted FPF as a valuable marker of mitochondrial dysfunction. For instance, an early study found that FPF was measurable in single cardiac cells that were subjected to hypoxia [62]. Later experiments showed that FPF increases with oxidative stress in myocardial ischemia-reperfusion injury, and is accompanied by reduced mitochondrial respiratory activity [63]. Research in brain tissues has also shown utility of FPF in the assessment of metabolic function and damage secondary to hypoxia [64].

In the retina, recent investigations have demonstrated that oxidative stress leads to increased FPF that correlates with decreased mitochondrial function and increased apoptosis. Elner et al. exposed human RPE cells to hydrogen peroxide and ceramide to induce oxidative stress and observed significant increases in FPF [11]. In contrast, co-treatment with the antioxidant $N$-acetylcysteine prevented FPF elevations. A later experiment replicated these findings, showing that hydrogen peroxide treatment increased RPE FPF in a time- and dose-dependent manner [14]. Moreover, FPF elevations preceded and were associated with decreased mitochondrial membrane potential and increased apoptosis. In neural retinal tissue, similar FPF elevations were seen, and were prevented by antioxidant co-treatment. Lastly, RPE cells co-cultured with monocytes displayed increased FPF that correlated with expression of pro-inflammatory cytokines. Taken together, these studies provide compelling evidence that retinal FPF can act as a biomarker of mitochondrial metabolic dysfunction that precedes apoptosis and structural damage.

\section{Development of a retinal metabolic imaging device}

The potential for retinal FPF to be measured in vivo led to the development of a novel imaging device (Table 1). Elner et al. presented the findings from the first generation device [60]. They performed over 50 modifications to a Zeiss F4 fundus camera (Carl Zeiss, Oberkochen, Germany), including the addition of $467 \mathrm{~nm}$ excitation and $535 \mathrm{~nm}$ emission filters, a back-illuminated, electron-multiplying, charge-coupled device camera, and an interfaced computer 
Table 1 Summary of retinal metabolic imaging devices.

\begin{tabular}{lllllll}
\hline Generation & Year & $\begin{array}{l}\text { FPF captured } \\
\text { field of view }\end{array}$ & $\begin{array}{l}\text { Captures } \\
\text { infrared image }\end{array}$ & Focus & $\begin{array}{l}\text { Scanning laser } \\
\text { ophthalmoscope }\end{array}$ & $\begin{array}{l}\text { Prior } \\
\text { publications }\end{array}$ \\
\hline First & 2008 & $3^{\circ}$ & No & Manual & No & 4 \\
Second & 2012 & $15^{\circ}$ & Yes & Manual & Yes & 3 \\
Third & 2018 & $23^{\circ}$ & Yes & $\begin{array}{l}\text { Manual and } \\
\text { automatic }\end{array}$ & Yes & 0 \\
\hline
\end{tabular}

$F P F$ flavoprotein fluorescence. with customized image capture and analysis software. This camera had a field of view of $3^{\circ}$.

Later, this group developed a second generation camera, which was a fully customized, proprietary device designed to enhance retinal FPF and minimize lens fluorescence [13]. This device utilized a scanning laser ophthalmoscope to capture a $30^{\circ}$ infrared fundus image and a $15^{\circ}$ FPF image using similar custom filters as the first device. Optical masks and a dichroic filter were used to couple blue light into the entrance aperture and green light out of the exit aperture. These apertures were physically separated to reduce contamination of retinal signal by lens fluorescence and light scatter.

The third and latest generation camera, the OcuMet Beacon (OcuSciences Inc., Ann Arbor, MI), incorporates a confocal infrared scanner and a metabolic detector designed to enhance wavelengths near the emission peak of FPF. The camera uses an excitation band-pass filter centered on $465 \mathrm{~nm}$ and an emission band-pass filter to maximize retinal FPF and minimize contamination from lipofuscin and other fluorophores. This camera is a scanning laser ophthalmoscope that captures a $60^{\circ}$ infrared image and a $19^{\circ} \mathrm{FPF}$ image using automatic focusing, and interfaces with customized image quality assessment and analysis software.

\section{Mitochondrial imaging in patients with retinal disease}

Seven peer-reviewed articles have described FPF imaging in patients (Table 2).

\section{First generation device studies}

Elner et al. first examined retinal FPF in patients with PTC [60]. Six patients with recently diagnosed, treatment-naive PTC were compared to six age-matched healthy control patients. Patients with PTC showed at least 25\% higher average FPF intensity and average curve width $(\mathrm{CW})$, a measure of variation of FPF intensity in the image, in their more clinically affected eye. Elevated $\mathrm{CW}$ was thought to reflect the fact that different cells were affected by the disease process to different degrees. In contrast, control patients had no significant FPF intensity or CW differences between eyes.

Two early studies investigated the relationship between DR and FPF. Field et al. evaluated 21 patients with diabetes and 21 healthy control patients, while Elner et al. evaluated 14 patients with diabetes and 7 controls [11, 12]. While controlling for age, each of these studies found that mean FPF intensity and CW was significantly elevated in patients with diabetes compared to controls. In addition, diabetic patients with DR exhibited higher FPF intensity and CW than diabetic patients without DR. In fact, FPF intensity better predicted DR status than did hemoglobin A1c (HbA1c) [12]. Single patient with advanced AMD, CSCR, and retinitis pigmentosa also exhibited higher FPF intensity than age-matched controls [11]. Notably, these studies were the first to demonstrate that FPF intensity, and potentially $\mathrm{CW}$, increase progressively with age in healthy control eyes, indicating that age must be adjusted for in studies of FPF. These authors proposed that FPF elevations in older patients may be explained by increasing physiologic apoptosis or some degree of lipofuscin accumulation with age.

The most recent first generation study examined patients with CSCR [18]. In each of three patients with unilateral CSCR who had a disease duration ranging from 1 week to 10 months, the affected eye exhibited greater FPF intensity than the fellow eye. CSCR-affected eyes also averaged 98\% higher FPF intensity than age-matched control eyes.

\section{Second generation device studies}

Field et al. examined retinal FPF in patients with different stages of non-exudative AMD [13]. Three eyes with nonexudative AMD, three eyes with non-exudative AMD and GA, and six control eyes were studied. Mean FPF intensity was significantly higher in AMD eyes versus controls, and was even higher in AMD eyes where GA was present. Furthermore, qualitative and quantitative FPF heterogeneity across the image was elevated in AMD eyes and correlated with size of the atrophic lesion.

Later, two FPF studies were performed in patients with glaucoma. Ritch et al. recruited 14 patients with glaucoma and conducted a randomized, double-masked trial of a novel combination supplement with antioxidant and 


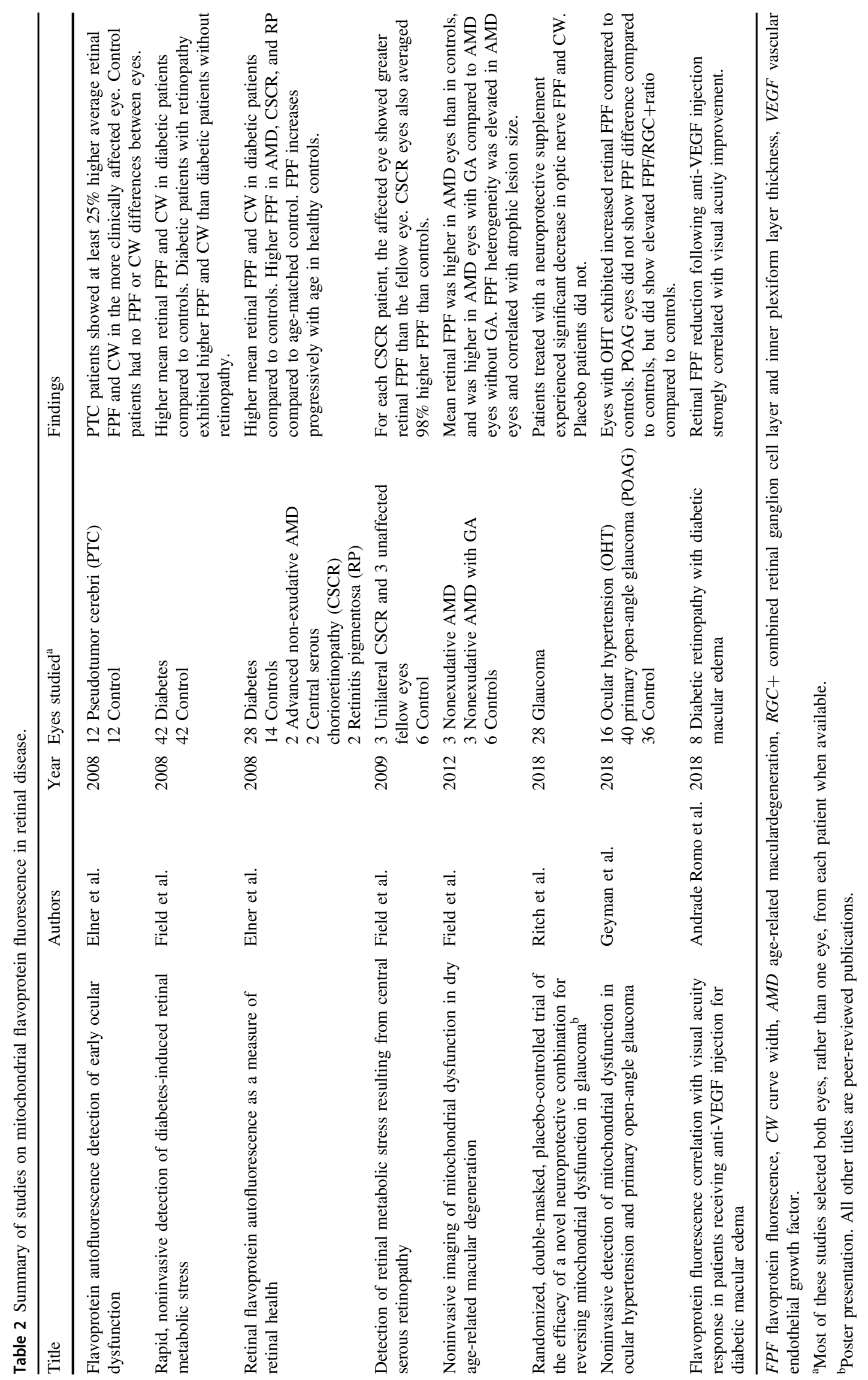


mitoprotective properties [17]. After 1 month, patients treated with the supplement experienced significant decreases in optic nerve FPF intensity and $\mathrm{CW}$, but patients treated with placebo did not. These findings suggested a reversal of mitochondrial dysfunction following neuroprotective supplementation. Afterward, Geyman et al. studied 16 eyes with ocular hypertension (OHT) and 40 with POAG [16]. They observed increased retinal FPF intensity in eyes with OHT compared to healthy control eyes. POAG eyes did not show a FPF difference compared to controls. However, after adjusting FPF intensity by RGC + inner plexiform layer thickness (RGC + ) to account for RGC atrophy, FPF/RGC + ratio was found to be elevated in POAG compared to controls. These findings suggested that mitochondrial dysfunction caused by OHT could be detected prior to clinically detectable changes or structural damage on optical coherence tomography (OCT). This study also considered that reduced numbers of mitochondria secondary to cell death may limit potential retinal FPF in advanced disease, and raised the question of how to account for this effect in future studies.

Most recently, Andrade Romo et al. assessed retinal FPF before and after anti-vascular endothelial growth factor (anti-VEGF) injection in patients with proliferative DR (PDR) and clinically significant diabetic macular edema [15]. In these eight patients, reductions in FPF intensity following injection were strongly correlated with VA improvements. In contrast, no significant correlation was observed between changes in central macular thickness and VA. This study demonstrated that FPF may be able to identify improvement in retinal metabolic function prior to detectable structural changes. Moreover, retinal FPF may serve as a tool to evaluate a patient's response to therapy.

\section{Third generation device study}

In the next section, the authors will present their findings using the third generation FPF imaging device, the OcuMet Beacon. A major limitation of the existing literature is an incomplete understanding of how baseline demographic and ocular characteristics influence FPF in healthy control eyes. The consequence of this is an absence of established healthy reference levels for FPF, which will be critical for the assessment of FPF in eyes with potential retinal disease. Prior imaging studies have also been limited to small samples. Lastly, while elevated FPF intensity and CW have been demonstrated in diabetes and DR, these parameters have not been compared among different clinical stages of DR. The aims of the authors' study were to establish a normative reference dataset of FPF for healthy eyes without vitreoretinal disease, to compare eyes with diabetes and DR to healthy eyes, and to compare FPF across stages of DR.

\section{Subjects and methods}

\section{Study design and participants}

An observational study was performed following Cleveland Clinic Institutional Review Board (IRB) approval. Each participant provided verbal and written informed consent prior to participation in the study. All study-related procedures were performed in accordance with good clinical practice (International Conference on Harmonization of Technical Requirements of Pharmaceuticals for Human Use [ICH] E6), applicable FDA regulations, the Health Insurance Portability and Accountability Act, and the Declaration of Helsinki.

Participants were patients aged 18 years or older seen at the Cleveland Clinic from August 6, 2018 to January 14, 2020. Control patients had no retinopathy. Diabetes patients had a documented diabetes diagnosis in the electronic medical record and no signs of retinopathy on dilated fundus examination performed by an optometrist or retinal specialist. DR patients had a clinical stage of DR that was classified at the discretion of a retina specialist. For analysis purposes, DR groups were defined as mild or moderate nonproliferative DR (NPDR) and PDR. Exclusion criteria were as follows: pseudophakia or aphakia, OHT or glaucoma, concomitant macular abnormalities or retinopathy or uveitis, hydroxychloroquine use, history of retinal detachment, vitreoretinal surgery in the past 3 months, and fluorescein angiogram on the day of imaging due to confounding of fluorescence signal. Only one eye per patient was included for analysis. In bilateral cases, the eye with higher quality images was selected. If image quality was similar, the eye was randomly selected.

For the control cohort, 347 eyes of 217 control patients were imaged. From this initial group, eyes were excluded from analysis for the following reasons: 91 eyes meeting exclusion criteria, 99 fellow eyes, and 6 eyes with inadequate image quality. The final control cohort consisted of 151 eyes from 151 patients. For the diabetes cohort, 480 eyes of 259 diabetic patients were imaged. Diabetic eyes were excluded for: 131 eyes meeting exclusion criteria, 220 fellow eyes, and 12 eyes with inadequate image quality. The final diabetes cohort consisted of 117 eyes from 117 patients.

\section{Retinal mitochondrial imaging}

The OcuMet Beacon, described in the section "Development of a Retinal Metabolic Imaging Device", was used for FPF imaging. Safety analysis performed on this device has determined that the infrared and blue light sources each deliver less than $1 \%$ of the maximum allowable energy levels designated by the International Electrotechnical 
Commission [65]. Both the device laser and light-emitting diode are therefore classified under the safest category, type I. Prior to imaging, eyes were dilated with $2.5 \%$ phenylephrine and $1.0 \%$ tropicamide and clinical fundus examination was performed. For each eye, at least two images were taken for quality control. The imaging process took no longer than $5 \mathrm{~min}$.

\section{Image processing}

Images were examined by the device operator and an image analysis expert at OcuSciences for quality metrics, including focus, centering on the macula, and shadow artefacts from eyelashes or eyelids. The higher quality image of the two was used for FPF quantification, or if the images were of similar quality, the mean value was taken. Eyes with no images of sufficient quality, or with occluding vitreous hemorrhage, were excluded from analysis.

Proprietary software was used to calculate FPF parameters for each image. For each eye, FPF intensity was calculated as the average FPF intensity over a $5.5-\mathrm{mm}$ diameter region of interest centered in the illumination area. FPF heterogeneity was calculated as the standard deviation of FPF intensity of all the pixels in the region of interest, following high-pass filtering to remove global gradients.

\section{Study variables}

From patient history, diabetes diagnosis, diabetes duration, and insulin use were determined. HbA1c within 3 months of the imaging encounter was collected if available. From clinical exam, VA, IOP, macular abnormalities (e.g., holes, vitreomacular traction, epiretinal membrane, drusen), concomitant retinopathy, and vitreous (e.g., posterior vitreous detachment (PVD)) were evaluated. If OCT was performed, Cirrus spectral domain high definition-OCT review software (V.9.5.1, Carl Zeiss Meditech, Dublin, CA) was used for calculation of central subfield thickness (CST). Clinically significant macular edema (CSMO) was determined by each clinician on dilated fundus exam according to Early Treatment Diabetic Retinopathy Study (ETDRS) criteria [66], and had the additional requirement of CST $\geq 250 \mu \mathrm{m}$.

\section{Statistical analysis}

SAS (Version 9.4, Cary, NC) was used for statistical analysis. Continuous variables were described as median [interquartile range], while categorical variables were described as frequency (percentage). VA was converted from Snellen numbers to ETDRS letters using the formula ETDRS $=85+50 \times \log _{10}$ (Snellen fraction) [67]. Prior to comparing FPF intensity between control and diabetes cohorts, greedy matching with the Euclidean distance algorithm was used [68], creating an age-matched dataset of 112 diabetic patients and 112 control patients, with a maximum difference of 3 years for any given match. Multivariable linear regression was used to model FPF intensity and FPF heterogeneity, and nomograms were created to depict these models. Multivariable linear regression with imputation for HbA1c was also used to model VA for the diabetic cohort. Regression coefficients were described as estimate [95\% confidence interval]. Analysis of variance was used to assess FPF differences across DR stages. $p<0.05$ were considered statistically significant.

\section{Results}

\section{Demographics and summary}

A total of 268 eyes were studied, which included 151 healthy control eyes and 117 diabetic eyes (Table 3). Overall, the median age was 63.5 [54.4, 69.5] years, and $129(48.1 \%)$ of patients were female. Race distribution was 164 (61.2\%) White, 84 (31.3\%) Black, and 20 (7.5\%) other. Smoking status was 117 (43.7\%) current, 58 (21.6\%) former, and 93 (34.7\%) never. Median IOP was 15.0 [13.0, 17.0] mmHg. PVD was present in 57 (21.3\%) eyes. Median CST was 261.0 [243.0, 286.0] $\mu \mathrm{m}$. Median VA was 80.2 [80.2, 85.0] ETDRS letters.

Control and diabetes cohorts were similar in terms of age, gender, eye laterality, IOP, and CST $(p \geq 0.18$ for each). Race distributions significantly differed between cohorts $(p<0.001)$, of which Black patients made up 17.9\% of controls and $48.7 \%$ of diabetics. Control eyes had significantly higher rates of PVD at $31.8 \%$ compared to $7.7 \%$ $(p<0.001)$. VA was significantly lower in diabetic eyes at 80.2 [76.2, 85.0] compared to 85.0 [80.2, 85.0] letters $(p<0.001)$.

Of the 117 patients with diabetes, 16 (13.7\%) had Type 1 and 101 (86.3\%) had Type 2 diabetes (Table 4). Median duration since diabetes diagnosis was $10.5[5.0,14.0]$ years, HbAlc was $7.0[6.3,7.8] \%$, and $51(43.6 \%)$ patients used insulin. On examination, 63 (53.8\%) had no retinopathy, 29 (24.8\%) had mild or moderate NPDR, and 25 (21.4\%) had PDR. Eight (6.8\%) patients had CSME.

\section{Healthy controls}

Median FPF intensity in control patients was $73.0[56.3,83.8]$ and median FPF heterogeneity was 0.51 [0.36, 0.67]. Representative infrared and FPF images of healthy control and diabetic eyes are shown in Fig. 4. Notably, FPF intensity was highly consistent between the two images taken for each patient when both images were of adequate quality. 
Table 3 Overall demographics and summary data.

\begin{tabular}{|c|c|c|c|c|}
\hline Factor & $\begin{array}{l}\text { Total } \\
(N=268)\end{array}$ & $\begin{array}{l}\text { Control } \\
(N=151)\end{array}$ & $\begin{array}{l}\text { Diabetes } \\
(N=117)\end{array}$ & $p$ value \\
\hline Age (years) & $63.5[54.4,69.5]$ & $63.5[53.3,69.5]$ & $63.5[54.5,69.3]$ & $0.45^{\mathrm{a}}$ \\
\hline \multicolumn{5}{|l|}{ Gender } \\
\hline Female & $129(48.1 \%)$ & $69(45.7 \%)$ & $60(51.3 \%)$ & \multirow[t]{2}{*}{$0.36^{\mathrm{b}}$} \\
\hline Male & $139(51.9 \%)$ & $82(54.3 \%)$ & $57(48.7 \%)$ & \\
\hline \multicolumn{5}{|l|}{ Race } \\
\hline White & $164(61.2 \%)$ & $110(72.8 \%)$ & $54(46.2 \%)$ & \multirow[t]{3}{*}{$<0.001^{\mathrm{b}}$} \\
\hline Black & $84(31.3 \%)$ & $27(17.9 \%)$ & $57(48.7 \%)$ & \\
\hline Other & $20(7.5 \%)$ & $14(9.3 \%)$ & $6(5.1 \%)$ & \\
\hline \multicolumn{5}{|l|}{ Smoking status } \\
\hline Current & $117(43.7 \%)$ & $12(7.9 \%)$ & $9(7.7 \%)$ & \multirow[t]{3}{*}{$0.17^{\mathrm{b}}$} \\
\hline Former & $58(21.6 \%)$ & $43(28.5 \%)$ & $46(39.3 \%)$ & \\
\hline Never & $93(34.7 \%)$ & $96(63.6 \%)$ & $62(53.0 \%)$ & \\
\hline \multicolumn{5}{|l|}{ Eye laterality } \\
\hline Right & $138(51.5 \%)$ & $73(48.3 \%)$ & $65(55.6 \%)$ & \multirow[t]{2}{*}{$0.24^{\mathrm{b}}$} \\
\hline Left & $130(48.5 \%)$ & $78(51.7 \%)$ & $52(44.4 \%)$ & \\
\hline Intraocular pressure $(\mathrm{mmHg})^{*}$ & $15.0[13.0,17.0]$ & $15.0[14.0,16.0]$ & $15.0[13.0,17.0]$ & $0.60^{\mathrm{a}}$ \\
\hline History of posterior vitreous detachment & $57(21.3 \%)$ & $48(31.8 \%)$ & $9(7.7 \%)$ & $<0.001^{\mathrm{b}}$ \\
\hline Central subfield thickness $(\mu \mathrm{m})^{*}$ & $261.0[243.0,286.0]$ & $266.0[256.0,284.0]$ & $247.5[236.0,291.0]$ & $0.18^{\mathrm{a}}$ \\
\hline Visual acuity (ETDRS letters) & $80.2[80.2,85.0]$ & $85.0[80.2,85.0]$ & $80.2[76.2,85.0]$ & $<0.001^{\mathrm{a}}$ \\
\hline
\end{tabular}

Continuous variables are described as Median [Q1, Q3]. Categorical variables are described as frequency (percentage). *Data not available for all patients. Missing values: intraocular pressure $=8$, central subfield thickness $=219$.

$p$ values: ${ }^{a}$ Kruskal-Wallis test, ${ }^{\text {b}}$ Pearson's chi-square test.

Statistically significant values are bolded and italicized.
Table 4 Diabetes cohort summary.

\begin{tabular}{ll}
\hline Factor & Total $(N=117)$ \\
\hline Diabetes diagnosis & \\
$\quad$ Type 1 & $16(13.7 \%)$ \\
$\quad$ Type 2 & $101(86.3 \%)$ \\
Diabetes duration (years) & $10.5[5.0,14.0]$ \\
Hemoglobin A1c (\%) & $7.0[6.3,7.8]$ \\
Insulin use & $51(43.6 \%)$ \\
Diabetic retinopathy stage & \\
$\quad$ None & $63(53.8 \%)$ \\
$\quad$ Mild-moderate nonproliferative & $29(24.8 \%)$ \\
$\quad$ Proliferative & $25(21.4 \%)$ \\
Clinically significant macular edema & $8(6.8 \%)$ \\
\hline
\end{tabular}

Continuous variables are described as Median [Q1, Q3]. Categorical variables are described as frequency (percentage). *Data not available for all subjects. Missing values: hemoglobin $\mathrm{A} 1 \mathrm{c}=24$, diabetes duration $=41$

Among control and diabetic eyes, median percent difference in FPF intensity between the first and second images was 1.7 $[1.0,3.5] \%$.

Multivariable regression analysis of FPF intensity showed that age and gender were significant predictors (Table 5). Older age was associated with higher FPF intensity $(+1.40[1.26,1.55]$ units/year, $p<0.001)$. Female gender was also associated with higher FPF intensity
$(+6.46[2.98,9.93]$ units, female vs. male, $p<0.001)$. Race, smoking status, eye laterality, IOP, and history of PVD were not predictive ( $p>0.16$ for each).

In multivariable regression analysis of FPF heterogeneity, age was the only significant predictor (Table 6). Older age was associated with higher FPF heterogeneity $(+0.01[0.00,0.01]$ units/year, $p<0.001)$. Gender, race, smoking status, eye laterality, IOP, and history of PVD were not predictive ( $p>0.10$ for each). To further examine the relationship between age and FPF, control patients were stratified by age subgroups in 10-year increments (Fig. 5), which confirmed the trend of increasing FPF intensity and FPF heterogeneity with age.

Based on the regression models, nomograms were constructed for control patients (Fig. 6). Using these nomograms, expected FPF intensity and FPF heterogeneity can be calculated for a healthy control patient by entering their demographic and ocular characteristics.

\section{Diabetes and DR}

Median FPF intensity in patients with diabetes was 76.0 $[67.0,92.0]$ and median FPF heterogeneity was $0.65[0.48$, 0.92]. Age-matched comparison with controls showed that in diabetic eyes, FPF intensity was a mean of $5.1[1.9,8.3]$ units higher $(p=0.002)$ and FPF heterogeneity was 0.15 $[0.07,0.24]$ units higher $(p<0.001)$ (Table 7). 

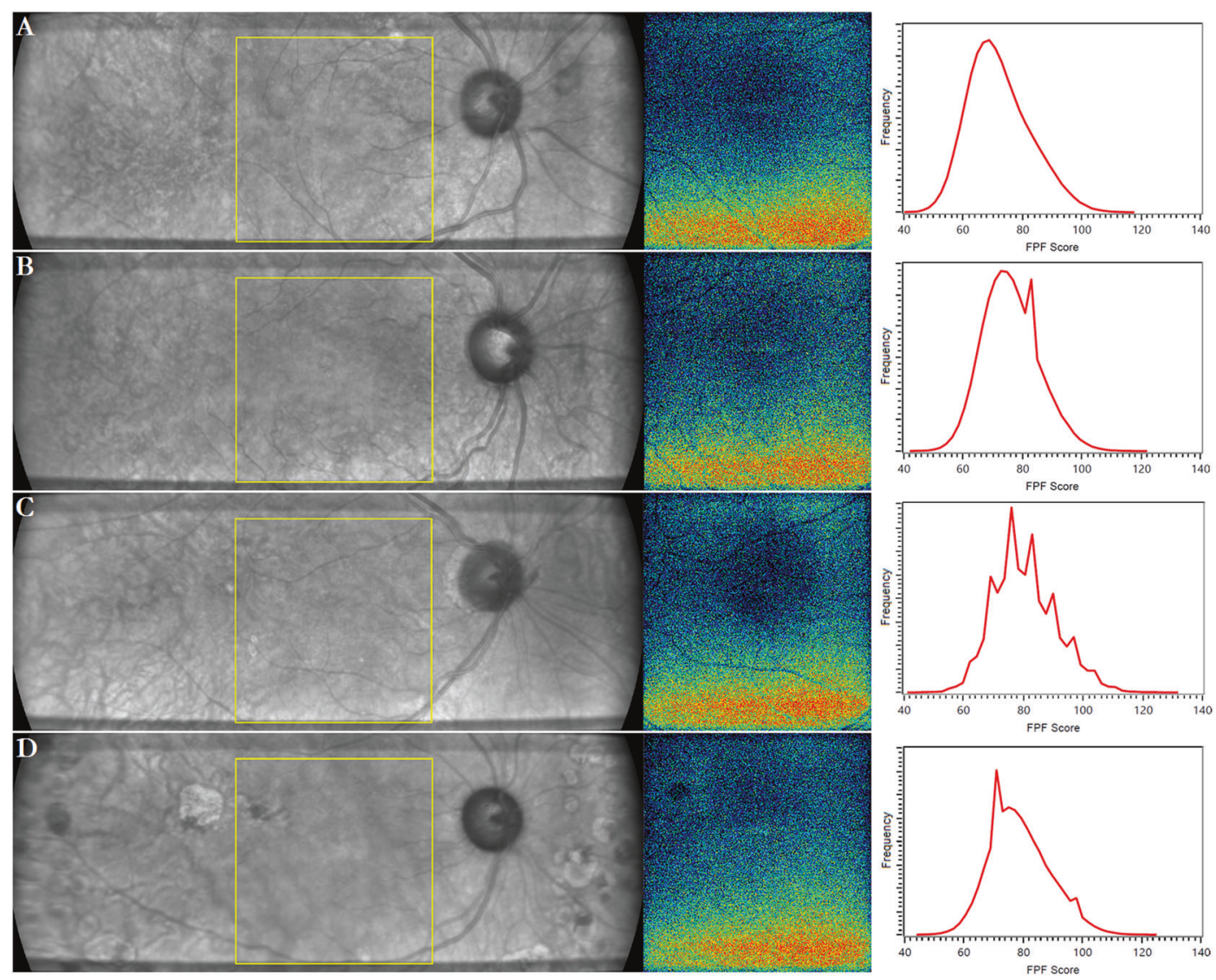

Age: 61 years

FPF intensity: 72.0

FPF heterogeneity: 0.26

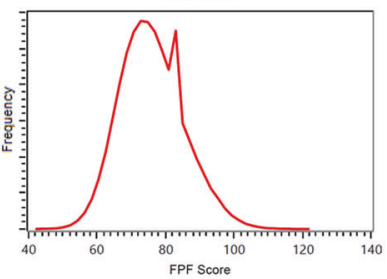

Age: 61 years

FPF intensity: 76.5

FPF heterogeneity: 0.58

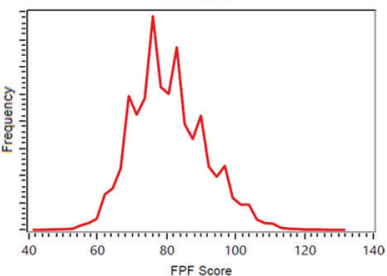

Age: 59 years

FPF intensity: 81.0

FPF heterogeneity: 0.32

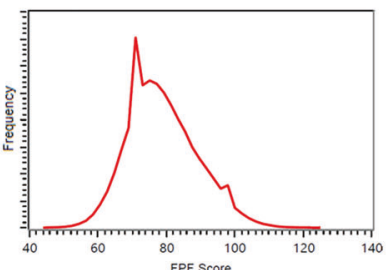

Age: 66 years

FPF intensity: 76.5

FPF heterogeneity: 0.44

Fig. 4 Infrared (IR) and flavoprotein fluorescence (FPF) images of healthy control and diabetic eyes. Representative images of a healthy control, b diabetes without retinopathy, c mild-moderate nonproliferative diabetic retinopathy, and $\mathbf{d}$ proliferative diabetic

Table 5 Multiple linear regression model for flavoprotein fluorescence intensity in control patients.

\begin{tabular}{lrrrr}
\hline Factor & Estimate & $95 \%$ CI & & $p$ value \\
\hline Intercept & -14.94 & -26.84 & -3.03 & 0.014 \\
Age (years) & 1.40 & 1.26 & 1.55 & $<\mathbf{0 . 0 0 1}$ \\
Gender: Female (vs Male) & 6.46 & 2.98 & 9.93 & $<\mathbf{0 . 0 0 1}$ \\
Race & & & & \\
$\quad$ Black (vs White) & -2.76 & -7.14 & 1.61 & 0.214 \\
$\quad$ Other (vs White) & 4.38 & -1.78 & 10.5 & 0.162 \\
Smoking status & & & & \\
$\quad$ Current (vs never) & 3.92 & -2.34 & 10.2 & 0.218 \\
$\quad$ Former (vs never) & 2.51 & -1.3 & 6.32 & 0.195 \\
Eye laterality: left (vs right) & 0.43 & -2.94 & 3.79 & 0.802 \\
Intraocular pressure (mmHg) & -0.17 & -0.74 & 0.4 & 0.556 \\
History of posterior vitreous & -2.32 & -6.04 & 1.38 & 0.218 \\
detachment & & & & \\
\hline Estimates denote the predict & & & &
\end{tabular}

Estimates denote the predicted change in flavoprotein fluorescence intensity for a 1 unit increase of a numeric factor, or for that factor level relative to reference level of a categorical factor, with other factors held constant.

Statistically significant values are bolded and italicized.

CI confidence interval. retinopathy. (Left) IR fundus image. The yellow box indicates the region where FPF is measured. (Center) FPF heat map. Warmer colors represent higher FPF intensities. (Right) Distribution of FPF intensities among all image pixels.

Table 6 Multiple linear regression model for flavoprotein fluorescence heterogeneity in control patients.

\begin{tabular}{|c|c|c|c|c|}
\hline \multirow{2}{*}{$\frac{\text { Factor }}{\text { Intercept }}$} & \multirow{2}{*}{$\begin{array}{l}\text { Estimate } \\
-0.03\end{array}$} & \multicolumn{2}{|l|}{$95 \% \mathrm{CI}$} & \multirow{2}{*}{$\frac{p \text { value }}{0.831}$} \\
\hline & & -0.33 & 0.27 & \\
\hline Age (years) & 0.01 & 0.00 & 0.01 & $<0.001$ \\
\hline Gender: female (vs male) & 0.00 & -0.08 & 0.09 & 0.930 \\
\hline \multicolumn{5}{|l|}{ Race } \\
\hline Black (vs White) & 0.00 & -0.11 & 0.11 & 0.956 \\
\hline Other (vs White) & -0.04 & -0.20 & 0.11 & 0.580 \\
\hline \multicolumn{5}{|l|}{ Smoking status } \\
\hline Current (vs never) & 0.05 & -0.11 & 0.21 & 0.511 \\
\hline Former (vs never) & 0.08 & -0.02 & 0.18 & 0.104 \\
\hline Eye laterality: left (vs right) & -0.03 & -0.12 & 0.05 & 0.485 \\
\hline Intraocular pressure $(\mathrm{mmHg})$ & 0.00 & -0.01 & 0.02 & 0.526 \\
\hline $\begin{array}{l}\text { History of posterior vitreous } \\
\text { detachment }\end{array}$ & 0.03 & -0.06 & 0.13 & 0.513 \\
\hline
\end{tabular}

Estimates denote the predicted change in flavoprotein fluorescence heterogeneity for a 1 unit increase of a numeric factor, or for that factor level relative to reference level of a categorical factor, with other factors held constant.

Statistically significant values are bolded and italicized.

$C I$ confidence interval. 
Fig. 5 Flavoprotein fluorescence (FPF) in healthy control eyes stratified by age. a FPF intensity and $\mathbf{b}$ FPF heterogeneity. Ends of boxes represent 1 st and 3rd quartiles, middle band represents median, "X" represents mean. Ends of whiskers represent minimum and maximum. Outliers, defined as $1.5 \times$ interquartile range beyond 1 st or 3 rd quartile, are shown as dots.
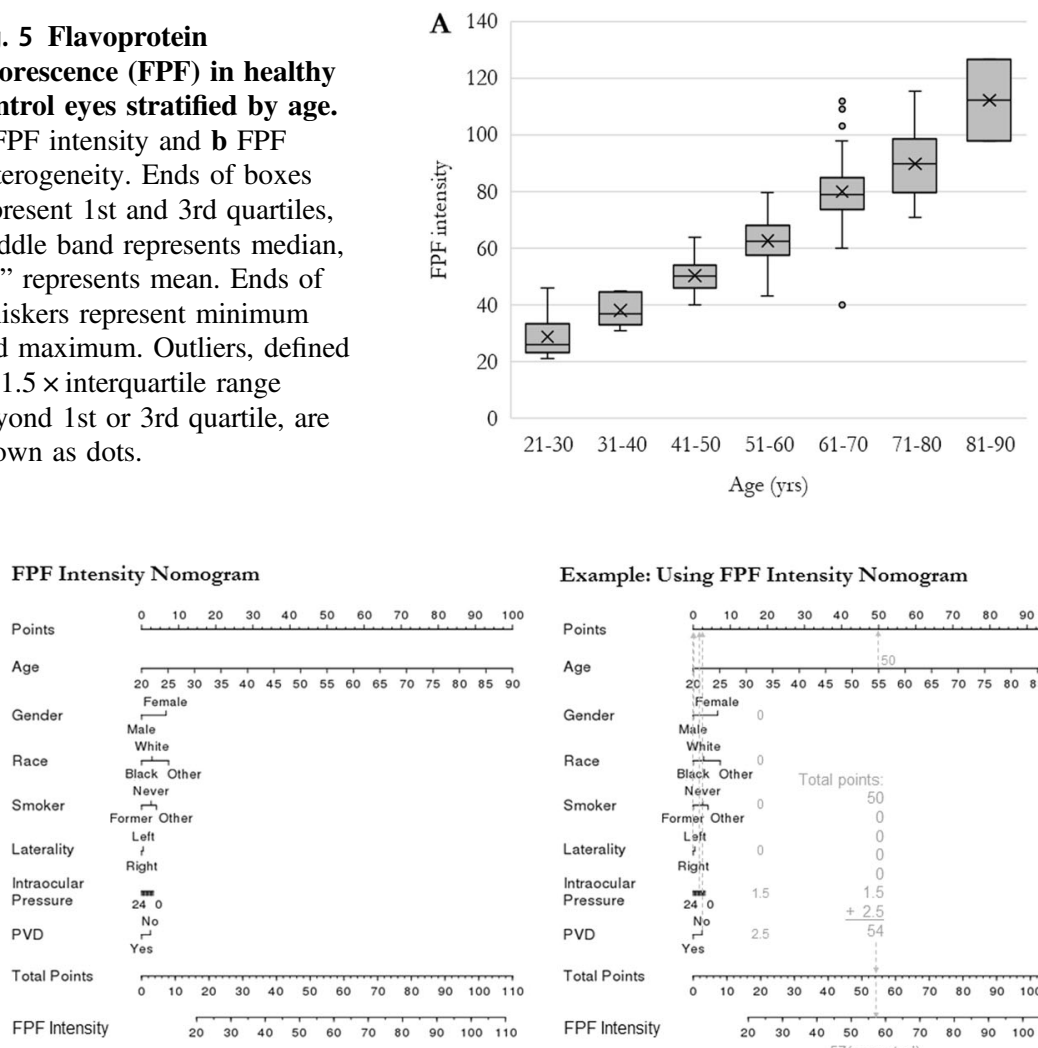

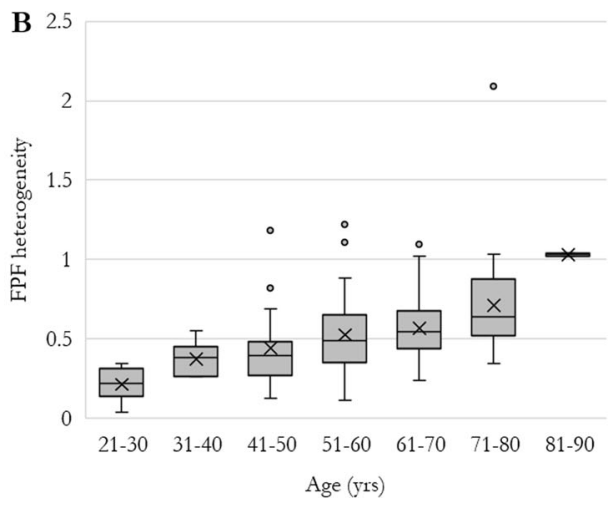

FPF Heterogeneity Nomogram

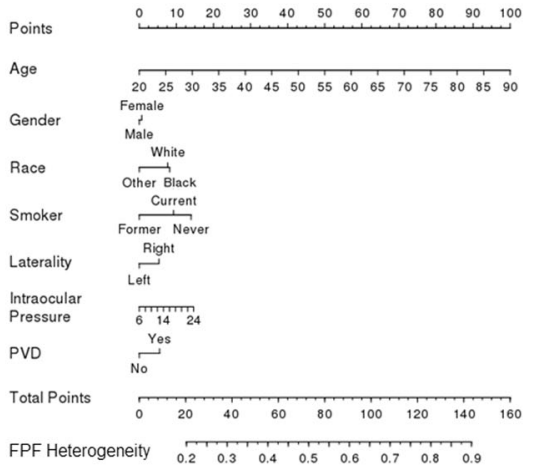

Fig. 6 Nomograms for flavoprotein fluorescence (FPF) intensity and FPF heterogeneity in healthy control patients. Based on these nomograms, demographic and ocular factors are used to calculate expected FPF intensity and FPF heterogeneity for a given patient. To use the nomogram, the number of points corresponding to each factor are added together, and the total points are mapped to the expected FPF value. For example, the center panel shows how the FPF intensity nomogram (left) would be used for a 55-year-old male, Black, former smoker whose right eye was imaged, and who has intraocular pressure of $12 \mathrm{mmHg}$ and no history of posterior vitreous detachment (PVD).
Table 7 Flavoprotein fluorescence in diabetic eyes compared to control eyes.

\begin{tabular}{llllr}
\hline Factor & $\begin{array}{l}\text { Mean difference } \\
\text { (diabetes vs. control) }\end{array}$ & $95 \%$ CI & $p$ value $^{\mathrm{a}}$ \\
\hline FPF intensity & 5.1 & 1.9 & 8.3 & $\mathbf{0 . 0 0 2}$ \\
FPF & 0.15 & 0.07 & 0.24 & $<\mathbf{0 . 0 0 1}$ \\
heterogeneity & & & & \\
\hline
\end{tabular}

Greedy matching with the Euclidean distance algorithm was used to create an age-matched dataset of 112 patients with diabetes and 112 control patients, with a maximum difference of 3 years for any given match. $F P F$ flavoprotein fluorescence, $C I$ confidence interval.

${ }^{a}$ Paired $t$-test.

Statistically significant values are bolded and italicized.

For the multivariable regression models in diabetic patients, size of the cohort limited analysis to twelve factors, which were chosen from those available. IOP, history of PVD, and eye laterality were not included because they showed no significant associations for controls. Diabetes duration and CST were not included due to relatively low data availability, and diabetes diagnosis was not included as
HbA1c and insulin use were thought to be more clinically important markers of glycaemic control [69]. Imputation was used for HbA1c, which had 24/117 missing values (Tables 8 and 9).

Significant predictors of higher FPF intensity were older age $(+1.25[1.01,1.49]$ units/year, $p<0.001)$ and PDR $(+9.66$ [3.07, 16.24] units, PDR vs no DR, $p=0.004)$. The only clearly significant predictor of lower FPF intensity was Black race $(-11.01[-16.01,-6.00]$ units, black vs White, $p<0.001$ ), while "other" race was of borderline significance $(-10.99$ [-22.00, 0.02] units, other vs White, $p=0.050)$. Gender, smoking status, HbA1c, insulin use, mild-moderate NPDR, and CSME were not predictive ( $p>0.11$ for each).

Significant predictors of higher FPF heterogeneity were older age $(+0.02[0.01,0.02]$ units/year, $p<0.001)$, insulin use $(+0.16[0.02,0.30]$ units, $p=0.031)$, and PDR $(+0.20$ $[0.03,0.38]$, PDR vs no DR, $p=0.023$ ). No other factors were predictive, including gender, race, smoking status, HbAlc, mild-moderate NPDR, and CSME ( $p>0.05$ for each).

To further evaluate associations between clinical DR stage and FPF, patients were stratified into age-matched subgroups of control, diabetes without DR, mild-moderate 
Table 8 Multivariable linear regression model for flavoprotein fluorescence intensity in patients with diabetes.

\begin{tabular}{lrrrr}
\hline Factor & Estimate & $95 \%$ CI & & $p$ value \\
\hline Intercept & 3.84 & -18.70 & 26.39 & 0.736 \\
Age (years) & 1.25 & 1.01 & 1.49 & $<\mathbf{0 . 0 0 1}$ \\
Gender: female (vs male) & 4.10 & -1.04 & 9.24 & 0.118 \\
Race & & & & \\
$\quad$ Black (vs White) & -11.01 & -16.01 & -6.00 & $<\mathbf{0 . 0 0 1}$ \\
$\quad-10.99$ & -22.00 & 0.02 & $\mathbf{0 . 0 5 0}$ \\
$\quad$ Other (vs White) & & & & \\
Smoking Status & 3.19 & -6.50 & 12.88 & 0.519 \\
$\quad$ Current (vs never) & -2.60 & -7.96 & 2.75 & 0.341 \\
$\quad$ Former (vs never) & -0.26 & -2.20 & 1.69 & 0.785 \\
Hemoglobin Alc (\%) & 0.70 & -4.70 & 6.10 & 0.800 \\
Insulin use & & & & \\
Diabetic retinopathy stage & & & & \\
$\quad$ Mild-moderate & 4.23 & -1.74 & 10.19 & 0.165 \\
nonproliferative & & & & \\
$\quad$ Proliferative & 9.66 & 3.07 & 16.24 & $\mathbf{0 . 0 0 4}$ \\
Clinically significant diabetic & 0.14 & -9.91 & 10.19 & 0.978 \\
macular edema & & & & \\
\hline Estmates & & &
\end{tabular}

Estimates denote the predicted change in flavoprotein fluorescence intensity for a 1 unit increase of a numeric factor, or for that factor level relative to reference level of a categorical factor, with other factors held constant.

CI confidence interval.

Statistically significant values are bolded and italicized.

NPDR, and PDR (Fig. 7). Median FPF intensities for these subgroups were $73.0[56.3,83.8], 76.0[62.0,86.0], 73.0$ $[68.5,95.0]$, and $73.0[69.3,88.8]$, respectively $(p=0.04)$. Median FPF heterogeneities were 0.51 [0.36, 0.67], 0.58 $[0.43,0.74], 0.85[0.61,1.00]$, and $0.90[0.51,1.20]$, respectively $(p<0.001)$.

Finally, the relationship between VA and FPF parameters in diabetic patients was assessed. First, univariable regression demonstrated that CSME, higher CST, and higher FPF heterogeneity were predictive of worse VA $(p \leq 0.004$ for each, Table 10). PDR was also predictive of worse VA compared to diabetic patients without DR $(p=0.003)$, but mild-moderate NPDR was not predictive $(p=0.491)$. Age, gender, diabetes diagnosis, diabetes duration, $\mathrm{HbA} 1 \mathrm{c}$, insulin use, and FPF intensity were not predictive of VA ( $p>0.28$ for each).

In the multivariable regression model of VA, diabetes duration and CST were removed due to relatively low data availability, but all other factors were included (Table 11). Higher FPF heterogeneity was a significant predictor of worse VA, with a regression coefficient of -13.23 [ -18.67 , $-7.79]$ letters/unit, $p<0.001$. No other factors in the model, including age, gender, diabetes diagnosis, $\mathrm{HbAlc}$, insulin use, DR stage, CSME, and FPF intensity, were predictive $(p \geq 0.060$ for each).
Table 9 Multivariable linear regression model for flavoprotein fluorescence heterogeneity in patients with diabetes.

\begin{tabular}{|c|c|c|c|c|}
\hline \multirow{2}{*}{$\frac{\text { Factor }}{\text { Intercept }}$} & \multirow{2}{*}{$\frac{\text { Estimate }}{-0.24}$} & \multicolumn{2}{|c|}{$95 \% \mathrm{CI}$} & \multirow{2}{*}{$\frac{p \text { value }}{0.409}$} \\
\hline & & -0.79 & 0.32 & \\
\hline Age (years) & 0.02 & 0.01 & 0.02 & $<0.001$ \\
\hline Gender: Female (vs Male) & 0.11 & -0.03 & 0.24 & 0.133 \\
\hline \multicolumn{5}{|l|}{ Race } \\
\hline Black (vs White) & -0.05 & -0.18 & 0.08 & 0.454 \\
\hline Other (vs White) & -0.04 & -0.34 & 0.25 & 0.767 \\
\hline \multicolumn{5}{|l|}{ Smoking Status } \\
\hline Current (vs Never) & -0.26 & -0.51 & 0.00 & 0.051 \\
\hline Former (vs Never) & -0.13 & -0.27 & 0.01 & 0.066 \\
\hline Hemoglobin A1c (\%) & -0.01 & -0.05 & 0.03 & 0.705 \\
\hline Insulin use & 0.16 & 0.02 & 0.30 & 0.031 \\
\hline \multicolumn{5}{|l|}{ Diabetic retinopathy stage } \\
\hline $\begin{array}{l}\text { Mild-moderate } \\
\text { nonproliferative }\end{array}$ & 0.15 & -0.01 & 0.31 & 0.059 \\
\hline Proliferative & 0.20 & 0.03 & 0.38 & 0.023 \\
\hline $\begin{array}{l}\text { Clinically significant diabetic } \\
\text { macular edema }\end{array}$ & 0.15 & -0.11 & 0.42 & 0.264 \\
\hline
\end{tabular}

Estimates denote the predicted change in flavoprotein fluorescence intensity for a 1 unit increase of a numeric factor, or for that factor level relative to reference level of a categorical factor, with other factors held constant.

Statistically significant values are bolded and italicized.

CI confidence interval.

\section{Discussion}

The purpose of the authors' study was to establish normative reference levels for retinal FPF in healthy eyes, to compare healthy eyes to eyes with diabetes and DR, and to compare FPF across stages of DR. In the current literature, healthy reference levels for FPF parameters, and the demographic and ocular factors that influence them, are not well established. From our data, we generated nomograms that estimate a healthy patient's expected FPF intensity and FPF heterogeneity based on demographic and ocular characteristics. Establishment of accurate, patient-specific reference levels using such tools will be essential to future use of this imaging modality, particularly in individuals with retinal disease. Moreover, in clinical practice, reference levels will be essential in determining which patients have an abnormal result and may need further evaluation.

While FPF intensity and CW have been reported to increase progressively with age $[11,12]$, few other factors have been associated with FPF parameters in healthy retinas. Supporting previous reports, we found that older age is independently associated with higher FPF intensity and heterogeneity in control patients. This suggests both higher mitochondrial dysfunction, and mitochondrial dysfunction affecting different retinal cells to varying degrees. 


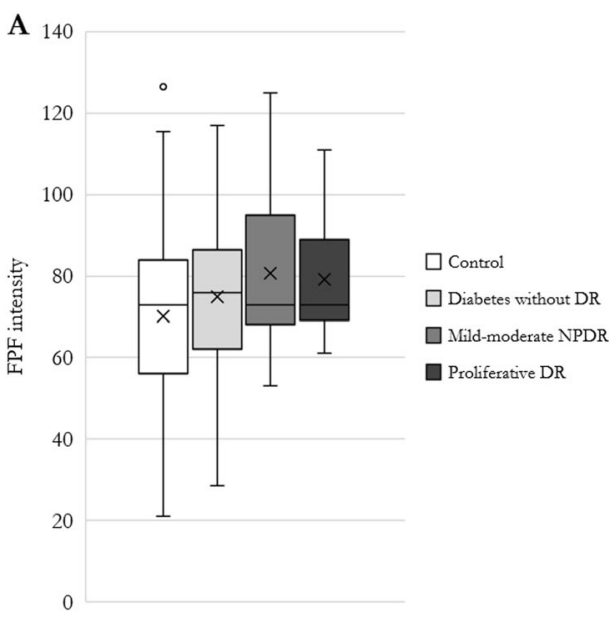

Fig. 7 Flavoprotein fluorescence (FPF) parameters in age-matched patients stratified by stage of diabetic retinopathy (DR). a FPF intensity and b FPF heterogeneity. In order to age-match by DR stage, some patients were not included in this analysis (ten patients with mild-moderate NPDR, six patients with proliferative DR). NPDR

Table 10 Univariable linear regression models for visual acuity in patients with diabetes.

\begin{tabular}{lrrrr}
\hline Factor & Estimate & $95 \%$ CI & & $p$ value \\
\hline Age (years) & 0.01 & -0.15 & 0.18 & 0.887 \\
Gender: female (vs male) & -1.03 & -4.63 & 2.57 & 0.571 \\
Diabetes diagnosis: Type 1 (vs & -0.71 & -5.94 & 4.53 & 0.790 \\
Type 2) & & & & \\
Diabetes duration (years)* & -0.09 & -0.26 & 0.08 & 0.286 \\
Hemoglobin A1c (\%)* & 0.04 & -1.11 & 1.18 & 0.950 \\
Insulin use & -1.53 & -5.14 & 2.09 & 0.406 \\
Diabetic retinopathy stage & & & & \\
$\quad$ Mild-moderate & -1.47 & -5.69 & 2.75 & 0.491 \\
nonproliferative (vs None) & & & & \\
$\quad$ Proliferative (vs None) & -6.88 & -11.32 & -2.43 & $\mathbf{0 . 0 0 3}$ \\
Clinically significant & -10.09 & -16.98 & -3.21 & $\mathbf{0 . 0 0 4}$ \\
macular edema & & & & \\
$\quad$ Central subfield thickness & -7.40 & -10.45 & -4.35 & $<\mathbf{0 . 0 0 1}$ \\
(100 s $\boldsymbol{\mu m}$ )* & & & & \\
FPF intensity & 0.00 & -0.10 & 0.09 & 0.967 \\
FPF heterogeneity & -10.29 & -14.42 & -6.16 & $<\mathbf{0 . 0 0 1}$ \\
\hline Estimas &
\end{tabular}

Estimates denote the predicted change in visual acuity (ETDRS letters) for a 1 unit increase of a numeric factor, or for that factor level relative to reference level of a categorical factor, with other factors held constant. *Data not available for all patients. Missing values: hemoglobin $\mathrm{A} 1 \mathrm{c}=24$, diabetes duration $=41$, Central subfield thickness $=83$.

Statistically significant values are bolded and italicized.

FPF flavoprotein fluorescence, $C I$ confidence interval.

Compared to patients aged $21-30$ years, those aged $81-90$ had median FPF intensity +86.3 units higher and median FPF heterogeneity +0.8 units higher. Interestingly, females were also seen to have higher FPF intensity, by a median of

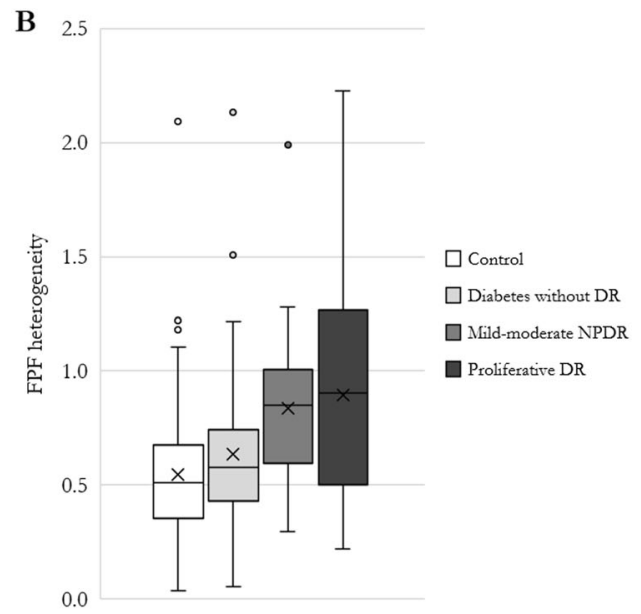

nonproliferative diabetic retinopathy. Ends of boxes represent 1st and 3rd quartiles, middle band represents median, " $X$ " represents mean. Ends of whiskers represent minimum and maximum. Outliers, defined as $1.5 \times$ interquartile range beyond 1 st or 3rd quartile, are shown as dots.

Table 11 Multivariable linear regression model for visual acuity in patients with diabetes.

\begin{tabular}{lrrrr}
\hline Factor & Estimate & $95 \%$ CI & & $p$ value \\
\hline Intercept & 83.76 & 68.81 & 98.71 & $<\mathbf{0 . 0 0 1}$ \\
Age (years) & 0.01 & -0.24 & 0.26 & 0.930 \\
Gender: female (vs male) & 0.53 & -3.35 & 4.42 & 0.785 \\
Diabetes diagnosis: Type 1 (vs & 4.11 & -1.80 & 10.03 & 0.170 \\
Type 2) & & & & \\
Hemoglobin A1c (\%) & -0.02 & -1.08 & 1.03 & 0.965 \\
Insulin use & 0.92 & -3.29 & 5.14 & 0.664 \\
Diabetic retinopathy stage & & & & \\
$\quad$ Mild/Moderate & 1.27 & -3.49 & 6.03 & 0.597 \\
nonproliferative (vs none) & & & & \\
$\quad$ Proliferative (vs none) & -5.29 & -10.79 & 0.22 & 0.060 \\
Clinically significant & -5.85 & -14.02 & 2.32 & 0.158 \\
macular edema & & & & \\
FPF intensity & 0.06 & -0.08 & 0.20 & 0.417 \\
FPF heterogeneity & -13.23 & -18.67 & -7.79 & $<\mathbf{0 . 0 0 1}$ \\
\hline
\end{tabular}

Estimates denote the predicted change in visual acuity (ETDRS letters) for a 1 unit increase of a numeric factor, or for that factor level relative to reference level of a categorical factor. *Data not available for all patients. Missing values: hemoglobin A1c $=24$.

Statistically significant values are bolded and italicized.

$F P F$ flavoprotein fluorescence, $C I$ confidence interval.

+6.46 units compared to males. Gender differences in FPF have not previously been reported. Race, smoking status, eye laterality, and history of PVD were not associated with FPF parameters. IOP, when within normal limits as in this cohort, was also not associated. While smoking increases oxidative stress and has been linked to oxidative damage in AMD, the effects of smoking in otherwise healthy patients 
may be too small to be reflected by FPF [70, 71]. Furthermore, smoking status was categorized fairly broadly in this study, and did not quantify smoking burden in terms of packs per day or pack-years. Racial differences in ocular oxygen metabolism have been described in some reports [72], but we did not observe any associations between race and FPF in controls.

To explain the higher FPF intensity observed in females, we look to literature regarding gender differences in retinal oxidative stress. In one study using a mouse model of neuronal ceroid lipofuscinosis, female mice were seen to exhibit higher oxidation rates in the retina, reflected by production of oxyradicals and lipid peroxides, when compared to age-matched male mice. Elevated caspase- 3 activation in female mice also indicated enhanced apoptosis [73]. In postmortem human eyes, higher levels of retinal iron have been observed in females at all ages [74], and gender differences have been reported in iron metabolism in both controls and patients with AMD [75]. Iron, which produces hydroxyl radicals via the Fenton reaction, is a prooxidant that has been implicated in various age-related diseases. Moreover, female gender is an established risk factor for AMD [76], a disease in which oxidative stress is heavily implicated. While the reasons for female predominance in AMD are not fully understood, previous research has pointed to post-menopausal loss of estrogen, which has protective antioxidant effects [77].

On the other hand, age has previously been demonstrated to have associations with FPF in smaller studies (see section "First generation device studies") [11, 12]. The authors of those studies proposed that FPF elevations may be due to increasing physiologic apoptosis or accumulation of lipofuscin with age. Another potential contributing factor, mentioned earlier, is retinal iron, which also increases with age. Higher retinal iron has been found in postmortem AMD-affected maculas compared to healthy maculas, and has been purported to induce oxidative stress in AMD $[74,78]$.

Another factor that could have greater influence is increasing fluorescence from fluorophores not in the retina, but in other ocular structures [79]. Lens and corneal fluorescence may play some role in the elevations of measured fluorescence with increasing age. While the novel optical pathway of the OcuMet Beacon is designed to enhance retinal FPF and minimize fluorescent signal received from the lens and cornea, potential signal contamination from these structures must be considered.

The crystalline lens contains tryptophan and nontryptophan fluorophores [80, 81]. Excitation and emission peaks for these fluorophores are 295 and $329 \mathrm{~nm}$, and 364-472 and $437-523 \mathrm{~nm}$, respectively. Thus, spectral curves of nontryptophan fluorophores exhibit some degree of overlap with those of flavoproteins. With increasing age of the lens, photo-oxidation and other metabolic processes lead to a greater degree of protein bonding and an increased proportion of water-insoluble protein [82]. Consequently, crystalline lens fluorescence increases with age, and has been shown to do so in a linear fashion [83]. In individuals with diabetes, lens fluorescence has been shown to increase faster than in healthy individuals and correlate with diabetes duration and $\mathrm{HbA} 1 \mathrm{c}$ [84].

Furthermore, cataract development, which increases with age, is associated with the generation of lipid fluorophores in the lens [85]. In early cataract stages, these fluorophores exhibit spectra in the ultraviolet and violet regions $($ excitation $=302-330$, emission $=411 \mathrm{~nm}$ ), while mature cataracts display an increase of fluorescence in the blue-green region (430-480 nm), approaching the emission region of flavoproteins. Another consideration is that while lens fluorescence increases with age, lens transmission decreases. During FPF imaging, this could reduce the amount of excitation light that actually reaches the retina, and in turn, artificially reduce the measured fluorescence.

In the cornea, endogenous fluorophores exist in the epithelium and endothelium. The primary fluorophores are the flavoproteins, NADH, and NADPH [86, 87]. NADH and NADPH fluoresce in the reduced state, and as stated previously, have an excitation peak near $300-360 \mathrm{~nm}$, far from that of flavoproteins. Glycosylated collagen has also been shown as a source of corneal fluorescence, with peak excitation at $370 \mathrm{~nm}$ and emission at $440 \mathrm{~nm}$, which also differs from flavoproteins. Aging and elevated circulating glucose in diabetes have been shown to increase the amount of glycosylated collagen in various nonocular tissues $[88,89]$. Furthermore, in eyes of patients with diabetes, corneal fluorescence has been linked to glycosylated collagen, and was found to be strongly associated with DR severity [90].

Thus, when imaging retinal FPF, the lens and cornea are potential sources of confounding fluorescence. The OcuMet Beacon and previous generations of the device have been designed with narrow band-pass filters and special optical pathways to maximize signal-to-noise ratio. However, some degree of signal contamination is possible, particularly in older individuals and those with diabetes due to associated upregulation of endogenous fluorophores. In addition, variability and age-related changes in lenticular transmission of excitation light is an important factor to consider.

Currently, lens transmission is also the main barrier to using a retinal metabolic imaging device in pseudophakic eyes. Compared to the native lens, intraocular lenses (IOLs) have different spectral properties that influence both the device excitation beam and the emission light received. This challenge is further complicated by the fact that there are many different IOL models, which have a wide range of spectral transmission properties [91]. Our group is currently 
working to develop FPF compensation factors that can be applied to different IOL models. Ultimately, IOL compensation will be needed for such a device to be used in routine clinical practice.

The second cohort we studied consisted of patients with diabetes and DR. In these patients, FPF intensity and heterogeneity were elevated compared to age-matched control eyes. Previous studies have reported similar findings (see section "First generation device studies") [11, 12]. These results suggest that increased oxidative stress and mitochondrial dysfunction are present and measurable in the retinal tissue of patients with diabetes, including those with and without clinically apparent DR.

In diabetic eyes, as for controls, older age was a significant predictor of higher FPF intensity and FPF heterogeneity. Interestingly, Black race, when compared to White, was a predictor of lower FPF intensity (a considerable -11 units lower on average), despite no racial differences being seen in controls. This association was independent of any other factor in the regression models, including HbAlc, insulin use, DR stage, or the presence of CSME, and suggests that Black diabetic patients exhibited less mitochondrial oxidative stress than White counterparts. This is somewhat unexpected, as previous studies have demonstrated that Black patients have higher prevalence of DR, and when DR is present, it tends to be more severe and have higher rates of visually significant complications [92, 93]. Future studies are needed to clarify this relationship.

Insulin use was also independently associated with higher FPF heterogeneity. Insulin is typically prescribed when behavioral modifications and oral medications are insufficient to control blood glucose, and its use has been predictive of poorer glycemic control [69]. However, HbAlc was not predictive of FPF in our analysis. This could be partially due to limited statistical power but may also reflect previous research showing that the relationship between glycemic control and cellular damage in DR is not totally straightforward. For example, initial worsening of DR has been observed following rapid improvement of glycemic control [94]. Furthermore, insulin has been demonstrated to stimulate production of VEGF, a signaling molecule that upregulates vascular permeability and neovascularization in DR [95].

Unlike in control patients, gender was not predictive of FPF in diabetic patients. Potentially, this could be due to gender differences in DR. Among diabetic patients, there is an evidence that males may have a higher prevalence and worse severity of DR [96]. However, the issue of gender differences in microvascular complications of diabetes has proven to be complex [97], and further FPF studies will be needed to clarify this association. As in controls, smoking status was not a predictor. CSME was also not associated with FPF. Notably, only 8 of 117 diabetic patients had
CSME, which may have limited the ability to detect a difference.

When comparing different stages of DR and adjusting for other factors, eyes with PDR had elevated FPF intensity and heterogeneity compared to diabetic eyes without DR. However, neither FPF parameter was significantly different between mild-moderate NPDR and those with diabetes alone. This could be explained by insufficient sensitivity to detect particularly small FPF differences in the context of noise from other sources of fluorescence. Alternatively, metabolic dysfunction in early stages of DR may not significantly differ from that seen in diabetes alone. Previously, Field et al and Elner et al. demonstrated higher FPF intensity and CW in diabetic eyes with DR (unspecified stage) compared to diabetic eyes without retinopathy (see section "First generation device studies"). Qualitatively, DR stage may show a stronger trend of increasing FPF heterogeneity than FPF intensity in agematched patients, as seen in Fig. 6. Furthermore, FPF heterogeneity was found to be a significant negative predictor of VA in diabetic patients, whereas FPF intensity was not predictive. Although previous studies have often focused on FPF intensity, these findings suggest that future investigations into the utility of FPF heterogeneity could be revealing.

Mitochondrial imaging currently has a number of limitations to address. Although retinal disease severity has been shown to influence FPF, the effect of systemic comorbidities, such as hypertensive control, inflammatory diseases, obesity, and blood lipid disorders, is unclear. Similarly, more data are needed to establish truly robust FPF reference levels for high sensitivity and specificity. Correcting for lens status remains a challenge. Longitudinal data over the course of retinal disease are sparse, so it is difficult to determine if FPF is better suited for assessing short-term changes such as responses to therapy, or longterm monitoring of chronic disease. Furthermore, without strong longitudinal data we cannot draw conclusions about the relative chronology of FPF, structural changes, and disease progression. While FPF elevations have been shown to occur prior to necrosis in vitro [14], this finding has not been demonstrated in vivo. Establishing such chronological relationships would support the value of FPF as a screening tool. Also, assessing FPF in advanced retinal disease, where there is cell death and reduced numbers of mitochondria, remains an area to explore. Previous studies have adjusted by measures of tissue thickness [16], but other solutions have not been presented. Lastly, FPF imaging technology itself could be improved. While FPF intensity can be measured en face, currently there is no way to differentiate FPF between retinal layers. If cross-sectional slices could be visualized as they are on other modalities, they could provide valuable information. 


\section{Conclusion}

Functional imaging of mitochondria is a promising tool for the evaluation of retinal disease. Whereas most current imaging modalities evaluate anatomic features, mitochondrial imaging distinguishes itself as a way to assess metabolic health of the retina. Previous studies have demonstrated its value in the assessment of DR, AMD, and other retinal diseases. Future investigations involving larger numbers of patients, longitudinal study designs, and compensation factors for lens status and tissue loss will be important to further enhance the utility of this imaging modality.

Funding AXC was a recipient of a National Institutes of Health T32 Institutional Training Grant and Research to Prevent Blindness Medical Student Eye Research Fellowship in support of this study.

\section{Compliance with ethical standards}

Conflict of interest AXC: NIH Institutional Training T32 Award (grant), RPB Medical Student Eye Research Fellowship (grant); CAR: Employee of OcuSciences; RK: Regeneron (personal fees); SS: Allergan (personal fees); KET: Zeiss (grant); RPS: Genentech/Roche (personal fees), Alcon/Novartis (personal fees), Apellis (grant), Graybug (grant), Zeiss (personal fees), Bausch + Lomb (personal fees), Ophthea (personal fees), Regeneron (personal fees). TFC, GLH, TEG, RR, INB, RE: None.

Publisher's note Springer Nature remains neutral with regard to jurisdictional claims in published maps and institutional affiliations.

\section{References}

1. Schrier SA, Falk MJ. Mitochondrial disorders and the eye. Curr Opin Ophthalmol. 2011;22:325-31.

2. Barot M, Gokulgandhi MR. Mitochondrial dysfunction in retinal diseases. Curr Eye Res. 2011;36:1069-77. MK

3. Monsalve M, Borniquel S, Valle I, Lamas S. Mitochondrial dysfunction in human pathologies. Front Biosci. 2007;12:1131-53.

4. Jin GF, Hurst JS, Godley BF. Hydrogen peroxide stimulates apoptosis in cultured human retinal pigment epithelial cells. Curr Eye Res. 2001;22:165-73.

5. Barak A, Morse LS, Goldkorn T. Ceramide: a potential mediator of apoptosis in human retinal pigment epithelial cells. Investig Ophthalmol Vis Sci. 2001;42:247-54.

6. Kannan R, Jin M, Gamulescu M-A, Hinton DR. Ceramideinduced apoptosis: role of catalase and hepatocyte growth factor. Free Radic Biol Med. 2004;37:166-75.

7. Zhang C, Baffi J, Cousins SW, Csaky KG. Oxidant-induced cell death in retinal pigment epithelium cells mediated through the release of apoptosis-inducing factor. J Cell Sci. 2003;116:1915-23.

8. Masuda T, Shimazawa M, Hara H. Retinal diseases associated with oxidative stress and the effects of a free radical scavenger (Edaravone). Oxid Med Cell Longev. 2017;2017:9208489.

9. Benson RC, Meyer RA, Zaruba ME, McKhann GM. Cellular autofluorescence-is it due to flavins? J Histochem Cytochem. 1979;27:44-8.

10. Chance B, Schoener B, Oshino R, Itshak F, Nakase Y. Oxidationreduction ratio studies of mitochondria in freeze-trapped samples.
NADH and flavoprotein fluorescence signals. J Biol Chem. 1979;254:4764-71.

11. Elner SG, Elner VM, Field MG, Park S, Heckenlively PH Jr. Retinal flavoprotein autofluorescence as a measure of retinal health. Trans Am Ophthalmol Soc. 2008;106:215-22.

12. Field M, Elner V, Puro D, Feuerman J, Musch D, Pop-Busui R, et al. Rapid, noninvasive detection of diabetes-induced retinal metabolic stress. Arch Ophthalmol 2008;126:934-8.

13. Field MG, Comer GM, Kawaji T, Petty HREV. Noninvasive imaging of mitochondrial dysfunction in dry age-related macular degeneration. Ophthalmic Surg Lasers. 2012;43:358-65.

14. Field MG, Yang D, Bian Z-M, Petty HR, Elner VM. Retinal flavoprotein fluorescence correlates with mitochondrial stress, apoptosis, and chemokine expression. Exp Eye Res. 2011;93: 548-55.

15. Andrade Romo JS, Lynch G, Liu K, Kim D, Jansen M, Field MG, et al. Flavoprotein fluorescence correlation with visual acuity response in patients receiving anti-VEGF injection for diabetic macular edema. Oxid Med Cell Longev. 2018;2018:3567306.

16. Geyman LS, Suwan Y, Garg R, Field MG, Krawitz BD, Mo S, et al. Noninvasive detection of mitochondrial dysfunction in ocular hypertension and primary open-angle glaucoma. J Glaucoma. 2018; 27:592-599.

17. Ritch R, Suwan Y, Rosen R, Gustavo De Moraes C. Randomized, double-masked, placebo-controlled trial of the efficacy of a novel neuroprotective combination for reversing mitochondrial dysfunction in glaucoma. Poster session presented at: Am. Glaucoma Soc. New York: 2018 p. 1-4.

18. Field MG, Elner VM, Park S, Hackel R, Heckenlively JR, Elner SG, et al. Detection of retinal metabolic stress resulting from central serous retinopathy. Retina. 2009;29:1162-6.

19. Chan DC. Mitochondria: dynamic organelles in disease, aging, and development. Cell. 2006;125:1241-52.

20. Kooragayala K, Gotoh N, Cogliati T, Nellissery J, Kaden TR, French S, et al. Quantification of oxygen consumption in retina ex vivo demonstrates limited reserve capacity of photoreceptor mitochondria. Invest Ophthalmol Vis Sci. 2015;56:8428-36.

21. Wei YH. Oxidative stress and mitochondrial DNA mutations in human aging. Proc Soc Exp Biol Med. 1998;217:53-63.

22. Liang F-Q, Godley BF. Oxidative stress-induced mitochondrial DNA damage in human retinal pigment epithelial cells: a possible mechanism for RPE aging and age-related macular degeneration. Exp Eye Res. 2003;76:397-403.

23. Jarrett SG, Lin H, Godley BF, Boulton ME. Mitochondrial DNA damage and its potential role in retinal degeneration. Prog Retin Eye Res. 2008;27:596-607.

24. Du Y, Miller CM, Kern TS. Hyperglycemia increases mitochondrial superoxide in retina and retinal cells. Free Radic Biol Med. 2003;35:1491-9.

25. Kowluru RA, Mishra M. Oxidative stress, mitochondrial damage and diabetic retinopathy. Biochim Biophys Acta. 2015;1852:2474-83.

26. Rains JL, Jain SK. Oxidative stress, insulin signaling, and diabetes. Free Radic Biol Med. 2011;50:567-75.

27. Trudeau K, Molina AJA, Guo W, Roy S. High glucose disrupts mitochondrial morphology in retinal endothelial cells: implications for diabetic retinopathy. Am J Pathol. 2010;177:447-55.

28. Li X, Zhang M, Zhou H. The morphological features and mitochondrial oxidative stress mechanism of the retinal neurons apoptosis in early diabetic rats. J Diabetes Res. 2014;2014:678123.

29. Wu M-Y, Yiang G-T, Lai T-T, Li C-J. The oxidative stress and mitochondrial dysfunction during the pathogenesis of diabetic retinopathy. Oxid Med Cell Longev. 2018;2018:3420187.

30. Roy S, Kern TS, Song B, Stuebe C. Mechanistic insights into pathological changes in the diabetic retina: implications for targeting diabetic retinopathy. Am J Pathol. 2017;187:9-19. 
31. Kanwar M, Chan P-S, Kern TS, Kowluru RA. Oxidative damage in the retinal mitochondria of diabetic mice: possible protection by superoxide dismutase. Invest Ophthalmol Vis Sci. 2007;48:3805-11.

32. Lin $\mathrm{H}, \mathrm{Xu} \mathrm{H}$, Liang F-Q, Liang $\mathrm{H}$, Gupta $\mathrm{P}$, Havey AN, et al. Mitochondrial DNA damage and repair in RPE associated with aging and age-related macular degeneration. Investig Ophthalmol Vis Sci. 2011;52:3521-9.

33. Feher J, Kovacs I, Artico M, Cavallotti C, Papale A, Balacco Gabrieli C. Mitochondrial alterations of retinal pigment epithelium in age-related macular degeneration. Neurobiol Aging. 2006;27: 983-93.

34. Shen JK, Dong A, Hackett SF, Bell WR, Green WR, Campochiaro PA. Oxidative damage in age-related macular degeneration. Histol Histopathol. 2007;22:1301-8.

35. Jones MM, Manwaring N, Wang JJ, Rochtchina E, Mitchell P, Sue CM. Mitochondrial DNA haplogroups and age-related maculopathy. Arch Ophthalmol. 2007;125:1235-40.

36. Udar N, Atilano SR, Memarzadeh M, Boyer DS, Chwa M, Lu S, et al. Mitochondrial DNA haplogroups associated with age-related macular degeneration. Investig Ophthalmol Vis Sci. 2009;50: 2966-74.

37. Nordgaard CL, Berg KM, Kapphahn RJ, Reilly C, Feng X, Olsen TW, et al. Proteomics of the retinal pigment epithelium reveals altered protein expression at progressive stages of age-related macular degeneration. Investig Ophthalmol Vis Sci. 2006;47:815-22.

38. Nordgaard CL, Karunadharma PP, Feng X, Olsen TW, Ferrington DA. Mitochondrial proteomics of the retinal pigment epithelium at progressive stages of age-related macular degeneration. Investig Ophthalmol Vis Sci. 2008;49:2848-55.

39. Piippo N, Korhonen E, Hytti M, Kinnunen K, Kaarniranta K, Kauppinen A. Oxidative stress is the principal contributor to inflammasome activation in retinal pigment epithelium cells with defunct proteasomes and autophagy. Cell Physiol Biochem. 2018;49:359-367.

40. Brown EE, DeWeerd AJ, Ildefonso CJ, Lewin AS, Ash JD. Mitochondrial oxidative stress in the retinal pigment epithelium (RPE) led to metabolic dysfunction in both the RPE and retinal photoreceptors. Redox Biol. 2019;24:101201.

41. Age-Related Eye Disease Study Research Group. A randomized, placebo-controlled, clinical trial of high-dose supplementation with vitamins $\mathrm{C}$ and $\mathrm{E}$, beta carotene, and zinc for age-related macular degeneration and vision loss: AREDS report no. 8. Arch Ophthalmol. 2001;119:1417-36.

42. Cangemi FE. TOZAL Study: an open case control study of an oral antioxidant and omega-3 supplement for dry AMD. BMC Ophthalmol. 2007;7:3.

43. Osborne NN. Mitochondria: Their role in ganglion cell death and survival in primary open angle glaucoma. Exp Eye Res. 2010; 90:750-7.

44. Abu-Amero KK, Morales J, Bosley TM. Mitochondrial abnormalities in patients with primary open-angle glaucoma. Investig Ophthalmol Vis Sci. 2006;47:2533-41.

45. Moreno MC, Campanelli J, Sande P, Sánez DA, Keller Sarmiento MI, Rosenstein RE. Retinal oxidative stress induced by high intraocular pressure. Free Radic Biol Med. 2004;37:803-12.

46. Ju W-K, Liu Q, Kim K-Y, Crowston JG, Lindsey JD, Agarwal N, et al. Elevated hydrostatic pressure triggers mitochondrial fission and decreases cellular ATP in differentiated RGC-5 cells. Investig Ophthalmol Vis Sci. 2007;48:2145-51.

47. McMonnies C. Reactive oxygen species, oxidative stress, glaucoma and hyperbaric oxygen therapy. J Optom. 2018;11:3-9.

48. Wolf C, Gramer E, Müller-Myhsok B, Pasutto F, Reinthal E, Wissinger B, et al. Evaluation of nine candidate genes in patients with normal tension glaucoma: a case control study. BMC Med Genet. 2009;10:91.
49. Ju W-K, Kim K-Y, Duong-Polk KX, Lindsey JD, Ellisman MH, Weinreb RN. Increased optic atrophy type 1 expression protects retinal ganglion cells in a mouse model of glaucoma. Mol Vis. 2010;16:1331-42.

50. Jiang D, Xiong G, Feng H, Zhang Z, Chen P, Yan B, et al. Donation of mitochondria by iPSC-derived mesenchymal stem cells protects retinal ganglion cells against mitochondrial complex I defect-induced degeneration. Theranostics. 2019;9:2395-2410.

51. Lienhart W-D, Gudipati V, Macheroux P. The human flavoproteome. Arch Biochem Biophys. 2013;535:150-62.

52. Ning X, Baoyu Q, Yuzhen L, Shuli S, Reed E, Li QQ. Neurooptic cell apoptosis and microangiopathy in KKAY mouse retina. Int J Mol Med. 2004;13:87-92.

53. Kunz WS. Spectral properties of fluorescent flavoproteins of isolated rat liver mitochondria. FEBS Lett. 1986;195:92-6.

54. Kuznetsov AV, Mayboroda O, Kunz D, Winkler K, Schubert W, Kunz WS. Functional imaging of mitochondria in saponinpermeabilized mice muscle fibers. J Cell Biol. 1998;140:1091-9.

55. Delori FC, Dorey CK, Staurenghi G, Arend O, Goger DG, Weiter JJ. In vivo fluorescence of the ocular fundus exhibits retinal pigment epithelium lipofuscin characteristics. Investig Ophthalmol Vis Sci. 1995;36:718-29.

56. Thomson AJ. Fluorescence spectra of some retinyl polyenes. J Chem Phys. 1969;51:4106-16.

57. Docchio F, Boulton M, Cubeddu R, Ramponi R, Barker PD. Agerelated changes in the fluorescence of melanin and lipofuscin granules of the retinal pigment epithelium: a time-resolved fluorescence spectroscopy study. Photochem Photobiol. 1991;54:247-53.

58. Schomacker KT, Frisoli JK, Compton CC, Flotte TJ, Richter JM, Nishioka NS, et al. Ultraviolet laser-induced fluorescence of colonic tissue: basic biology and diagnostic potential. Lasers Surg Med. 1992;12:63-78.

59. Gopalkrishnapillai B, Nadanathangam V, Karmakar N, Anand S, Misra A. Evaluation of autofluorescent property of hemoglobinadvanced glycation end product as a long-term glycemic index of diabetes. Diabetes. 2003;52:1041-6.

60. Elner VM, Park S, Cornblath W, Hackel R, Petty HR. Flavoprotein autofluorescence detection of early ocular dysfunction. Arch Ophthalmol. 2008;126:259-60.

61. Choudhry N, Giani A, Miller JW. Fundus autofluorescence in geographic atrophy: a review. Semin Ophthalmol. 2010;25:206-13.

62. Koke JR, Wylie W, Wills M. Sensitivity of flavoprotein fluorescence to oxidative state in single isolated heart cells. Cytobios 1981;32:139-45.

63. Ranji M, Kanemoto S, Matsubara M, Grosso MA, Gorman JH, Gorman RC, et al. Fluorescence spectroscopy and imaging of myocardial apoptosis. J Biomed Opt. 2006;11:064036.

64. Chisholm KI, Ida KK, Davies AL, Papkovsky DB, Singer M, Dyson A, et al. In vivo imaging of flavoprotein fluorescence during hypoxia reveals the importance of direct arterial oxygen supply to cerebral cortex tissue. Adv Exp Med Biol. 2016;876:233-9.

65. Safety of laser products-Part 1: equipment classification and requirements. In: IEC 60825-1. Geneva: International Technical Commission; 2007.

66. Early Treatment Diabetic Retinopathy Study Research Group. Photocoagulation for diabetic macular edema. Arch Ophthalmol. 1985; 103:1796.

67. Gregori NZ, Feuer W, Rosenfeld PJ. Novel method for analyzing snellen visual acuity measurements. Retina. 2010;30:1046-50.

68. Avis D, Davis B, Steele JM. Probabilistic analysis of a greedy heuristic for euclidean matching. Probab Eng Inf Sci. 1988;2: 143-56.

69. Benoit SR, Fleming R, Philis-Tsimikas A, Ji M. Predictors of glycemic control among patients with Type 2 diabetes: a longitudinal study. BMC Public Health. 2005;5:36. 
70. Fearon MI, Phillips G, Carr T, Taylor M, Breheny D, Faux PS. The role of oxidative stress in smoking-related diseases. Mini Rev Org Chem. 2011;8:360-71.

71. Cano M, Thimmalappula R, Fujihara M, Nagai N, Sporn M, Wang AL, et al. Cigarette smoking, oxidative stress, the antioxidant response through $\mathrm{Nrf} 2$ signaling, and age-related macular degeneration. Vis Res. 2010;50:652-64.

72. Siegfried CJ. Racial differences in ocular oxidative metabolism. Arch Ophthalmol. 2011;129:849.

73. Guarneri R, Russo D, Cascio C, D’Agostino S, Galizzi G, Bigini P, et al. Retinal oxidation, apoptosis and age- and sex-differences in the mnd mutant mouse, a model of neuronal ceroid lipofuscinosis. Brain Res. 2004;1014:209-20.

74. Hahn P, Ying G, Beard J, Dunaief JL. Iron levels in human retina: sex difference and increase with age. Neuroreport. 2006;17:1803-6.

75. Čolak E, Žorić L, Radosavljević A, Ignjatović S. The association of serum iron-binding proteins and the antioxidant parameter levels in age-related macular degeneration. Curr Eye Res. 2018;43:659-65.

76. Age-Related Eye Disease Study Research Group. Risk factors associated with age-related macular degeneration. Ophthalmology. 2000;107:2224-32.

77. Kaarniranta K, Machalińska A, Veréb Z, Salminen A, Petrovski G, Kauppinen A. Estrogen signalling in the pathogenesis of agerelated macular degeneration. Curr Eye Res. 2015;40:226-33.

78. Hahn P. Maculas affected by age-related macular degeneration contain increased chelatable iron in the retinal pigment epithelium and Bruch's membrane. Arch Ophthalmol. 2003;121:1099.

79. Rovati L, Docchio F. Autofluorescence methods in ophthalmology. J Biomed Opt. 2004;9:9-21.

80. Jacobs R, Krohn DL. Fluorescence intensity profile of human lens sections. Investig Ophthalmol Vis Sci. 1981;20:117-20.

81. Bessems GJ, Keizer E, Wollensak J, Hoenders HJ. Non-tryptophan fluorescence of crystallins from normal and cataractous human lenses. Investig Ophthalmol Vis Sci. 1987;28:1157-63.

82. Van Best JA, Kuppens EV. Summary of studies on the blue-green autofluorescence and light transmission of the ocular lens. J Biomed Opt. 1996;1:243-50.

83. Siik S, Airaksinen PJ, Tuulonen A, Alanko HI, Nieminen H. Lens autofluorescence in healthy individuals. Acta Ophthalmol. 1991; 69:187-92.

84. Bleeker JC, van Best JA, Vrij L, van der Velde EA, Oosterhuis JA. Autofluorescence of the lens in diabetic and healthy subjects by fluorophotometry. Investig Ophthalmol Vis Sci. 1986;27:791-4.
85. Babizhayev MA. Lipid fluorophores of the human crystalline lens with cataract. Graefes Arch Clin Exp Ophthalmol. 1989;227:384-91.

86. Docchio F, Biancardi L. EUROEYE atlas of ocular fluorophores. Coimbra: Gráfica de Coimbra; 1995.

87. Udenfrend S. Fluorescence assays in biology and medicine. Volume II. New York, NY: Academic Press; 1969.

88. Odetti PR, Borgoglio A, Rolandi R. Age-related increase of collagen fluorescence in human subcutaneous tissue. Metabolism. 1992;41:655-8.

89. Monnier VM, Kohn RR, Cerami A. Accelerated age-related browning of human collagen in diabetes mellitus. Proc Natl Acad Sci USA. 1984;81:583-7.

90. Stolwijk TR, van Best JA, Oosterhuis JA, Swart W. Corneal autofluorescence: an indicator of diabetic retinopathy. Investig Ophthalmol Vis Sci. 1992;33:92-7.

91. van Norren D, van de Kraats J. Spectral transmission of intraocular lenses expressed as a virtual age. Br J Ophthalmol. 2007; 91:1374-5.

92. Emanuele N, Sacks J, Klein R, Reda D, Anderson R, Duckworth W, et al. Ethnicity, race, and baseline retinopathy correlates in the veterans affairs diabetes trial. Diabetes Care. 2005;28:1954-8.

93. Barsegian A, Kotlyar B, Lee J, Salifu M, McFarlane S. Diabetic retinopathy: focus on minority populations. Int J Clin Endocrinol Metab. 2017;3:34-45.

94. Feldman-Billard S, Larger É, Massin P. Early worsening of diabetic retinopathy after rapid improvement of blood glucose control in patients with diabetes. Diabetes Metab. 2018;44:4-14.

95. reins C, Giorgetti-Peraldi S, Murdaca J, Semenza GL, Van Obberghen E. Insulin stimulates hypoxia-inducible factor 1 through a phosphatidylinositol 3-kinase/target of rapamycindependent signaling pathway. J Biol Chem. 2002;277:27975-81.

96. Cherchi S, Gigante A, Contini P, Pistis D, Pilosu RM, Spanu MA, et al. Male type 2 diabetic patients have higher diabetic retinopathy prevalence. Diabetes. 2018;67:599-P.

97. Seghieri G, Policardo L, Anichini R, Franconi F, Campesi I, Cherchi S, et al. The effect of sex and gender on diabetic complications. Curr Diabetes Rev. 2017;13:148-60.

98. Md. Islam S, Honma M, Nakabayashi T, Kinjo M, Ohta N. $\mathrm{pH}$ dependence of the fluorescence lifetime of FAD in solution and in cells. Int J Mol Sci. 2013;14:1952-63.

99. Sparrow JR, Duncker T. Fundus autofluorescence and RPE lipofuscin in age-related macular degeneration. J Clin Med. 2014;3:1302-21. 